\title{
Combinatorial Design of a Nanobody that Specifically Targets Structured RNAs
}

\author{
F. Cawez ${ }^{1, \dagger}$, E. Duray ${ }^{1, \dagger}$, Y. $\mathrm{Hu}^{2}$, J. Vandenameele ${ }^{3}$, E. Romão ${ }^{2}$, C. Vincke ${ }^{2}$, \\ M. Dumoulin ${ }^{3}$, M. Galleni ${ }^{1}$, S. Muyldermans ${ }^{2}$ and M. Vandevenne ${ }^{1}$ \\ 1 - InBioS, Center for Protein Engineering, Biological Macromolecules and Biochemistry, Department of Life Sciences, University of Liege, \\ B4000 Sart Tilman, Belgium \\ 2 - Department of Cellular and Molecular Immunology, Vrije Universiteit Brussel, B-1050 Brussels, Belgium \\ 3 - InBioS, Center for Protein Engineering, Protein Folding and Enzymology, Department of Life Sciences, University of Liege, \\ B4000 Sart Tilman, Belgium
}

Correspondence to M. Galleni and M. Vandevenne: mgalleni@uliege.be; mvandevenne@uliege.be https://doi.org/10.1016/j.jmb.2018.03.032

Edited by Skerra Arne

\begin{abstract}
Recent advances in transcriptome sequencing and analysis have revealed the complexity of the human genome. The majority $(\approx 98 \%)$ of cellular transcripts is not translated into proteins and represents a vast, unchartered world of functional non-coding RNAs. Most of them adopt a well-defined three-dimensional structure to achieve their biological functions. However, only very few RNA structures are currently available which reflects the challenges associated with RNA crystallization. Nevertheless, these structures would represent a critical step in understanding functions of non-coding RNAs and their molecular mechanisms in the cell. The overall goal of this study is to develop an innovative and versatile tool to facilitate the functional study and crystallization of structured RNAs (stRNAs). In this work, we have engineered an antibody fragment from camelid heavy-chain antibody (nanobody) able to specifically bind with low nanomolar affinity to stRNA, while no binding could be detected for single-stranded DNA/RNA, double-stranded DNA/RNA or a negatively charged protein. However, this nanobody recognizes different and non-related stRNAs, this observation suggests that it binds to an epitope shared by these stRNAs. Finally, our data also show that the binding of the nanobody does not alter the secondary structure content of the stRNA as well as its unfolding/refolding processes during heat treatment. This work constitutes a successful proof of concept demonstrating that nanobodies can be engineered to recognize RNA-related epitopes.
\end{abstract}

(C) 2018 Elsevier Ltd. All rights reserved.

\section{Introduction}

The recent discovery that most of the human transcriptome is not translated into proteins is probably one of the most important observations of this decade [1]. Deciphering the function(s) of these non-proteincoding transcripts represents one of the major challenges that molecular biology is facing nowadays. The biological relevance of these transcripts, named noncoding RNAs (ncRNAs), for the biology of the cell is becoming increasingly evident $[2,3]$. Numerous classes of ncRNAs are highly expressed in adult and in developing tissues, and they often exhibit patterns of expression that are regulated precisely in time and space. These observations suggest that ncRNAs play key roles in the cell fate and in the development of various tissues [4,5], and therefore, the deregulation of their expression is associated with multiple pathologies such as cancers, neurological disorders and type 1 and 2 diabetes [6,7]. Most ncRNAs adopt a complex threedimensional (3D) structure to accomplish their biological functions [8,9]. Like proteins, RNAs present different organization levels to form their 3D structures [10]. The first level of organization consists in the nucleotidic sequence (primary structure). In the presence of salt, RNAs fold on themselves via Watson-Crick base pairing to form segments of double-stranded helices (hairpins) interrupted by single-stranded regions (loops). These secondary structure elements constitute the second level of 
organization. Finally, the hairpins and unpaired regions are precisely organized in space to form the third organization level. This tertiary structure, formed in vivo and in vitro, is most of the time dependent both on the presence of divalent ions, such as $\mathrm{Mg}^{2+}$, and the temperature. The biological functions of RNAs are mediated by their 3D structure [11-13].

Despite the increasing interest of the scientific community in these ncRNAs, only very few of them have been fully characterized to date. In this context, proteins engineered to specifically bind ncRNAs should allow their functions to be interrogated. Recently, this principle has been successfully applied in the case of engineered proteins that bind to single-stranded RNAs (ssRNAs). In particular, the most promising works so far were obtained with the Pumilio (PUF) proteins [14]. Engineered PUF proteins have been combined with various effector domains (GFP, PIN RNA endonuclease, RS-rich domains, GLD2 and CAF1 domains...) to monitor RNA localization, modulate splicing, generate site-specific RNA endonucleases and affect the translation and the stability of specific mRNAs in living cells $[15,16]$. These data suggest that fusing RNAbinding proteins (RBPs) to functional modules harboring particular activities is a successful approach to investigate target RNAs in the cell. In contrast, the design of proteins able to bind structured RNA (stRNA) remains poorly explored and this is mostly due to the lack of knowledge regarding the 3D structures of RNAs. Indeed, currently, the number of available RNA 3D structures represents less than $1 \%$ of all the structures reported in the Protein Data Bank (PDB) which reflects the difficulties associated with RNA crystallization: (i) RNAs harbor negatively charged phosphate groups that create repulsive forces that are disfavorable for crystal contacts [17]; (ii) RNAs often present flexible regions that lead to a conformational heterogeneity and finally [18]; and (iii) phase solving techniques of RNA crystals can be complex and laborious [19].

This work is focused on a particular stRNA transcribed from a pseudogene of the murine brain cytoplasmic RNA 1 (BC1), that we named $\varphi B C 1$ (NCBI Gene ID: 12031) and was thought to be the "real" BC1 gene for a long time. Indeed, it is now well established that most pseudogenes are transcribed into functional RNAs and appear to play important regulatory roles [20]; this is why we were interested in the functional and structural analysis of $\varphi B C 1 . B C 1$, its cousin RNA, is a murine 154-nt-long stRNA expressed in neurons; its human functional analog $(B C 200)$ is deregulated in the brain of patients with Alzheimer's disease [21]. $B C 1$ was shown to bind to translational regulators (elF4A, elF4B and PABP) and repress translation initiation [22]. $B C 1$ has been well characterized: its secondary structure content has been experimentally determined [23] and has revealed that the $5^{\prime}$ region forms a long hairpin, the central poly $A$ region is unpaired and the $3^{\prime}$ domain forms a second but smaller hairpin as shown in Fig. 1 .
Its molecular mechanisms are also well characterized; $B C 1$ represses translation through its central polyA and $3^{\prime}$ domains by blocking the catalytic activity of elF4A, an ATP-dependent RNA helicase, while stimulating its ATPase activity [22]. In contrast, barely any study was performed on $\varphi B C 1$. This 167-nt-long stRNA mostly differs from $B C 1$ in the central polyA and $3^{\prime}$ domains. Therefore, $\varphi B C 1$ lacks these two functional domains and consequently is predicted not to bind to translational regulators. This observation explains its attributed terminology as a pseudogene.

Engineering proteins able to bind to stRNAs (stRBPs) would have multiple applications. First, these stRBPs can be fused to different functional domains in order to investigate the biological function(s) of the targeted RNA in living cells or animals, similarly to what has been discussed above with PUF proteins $[15,24]$. Second, stRBPs can be engineered in order to inhibit the biological activity of catalytic RNAs, which are generally named ribozymes. Third, stRBPs can be used as crystallization aids to determine the 3D structure of the targeted RNAs. Indeed, a stRBP bound to RNA can increase the crystal contact surfaces and neutralize some of the negative charges that prevent efficient RNA crystal formation. Upon binding, the stRBP can also reduce the RNA flexibility. Moreover, stRBP structure can be used to solve the phase of RNA crystal by molecular replacement [25]. Finally, stRBPs constitute potential new therapeutic agents given that many stRNAs were shown to be associated with important pathologies [26].

The variable domains of heavy-chain antibodies, which are devoid of light chains, constitute a protein scaffold of choice to engineer proteins that bind specifically to stRNA. These antibody fragments, named VHHs or nanobodies, consist in the $\mathrm{N}$ terminal antigen-binding domain that is connected via the hinge region to the two constant domains $(\mathrm{CH} 2$ and $\mathrm{CH} 3$ ) in heavy-chain antibodies [27]. Nanobodies present several advantages compared to other antibodies or antibody fragments. First, they are much smaller (12-15 kDa) compared to conventional IgG (150 kDa) or antibody fragments (e.g., $\approx 50 \mathrm{kDa}$ for Fabs) while recognizing the cognate antigen with high affinity and specificity. In contrast to the $\mathrm{VH}-\mathrm{VL}$ pairing in Fabs, nanobodies are strictly monomeric and do not necessitate domain pairing to associate with antigen. In addition, nanobodies are more soluble and stable than other antigen-binding domains [28]. They are also poorly immunogenic in human and much easier to express and to purify from microbial hosts [28]. Furthermore, nanobodies present extended antigenbinding regions [especially complementary determining region 3 (CDR3)] that can penetrate into the catalytic site of enzymes, and potentially inhibit the enzymatic activity [29-31]. Finally, nanobodies have been successfully used to trace their antigen within eukaryotic cells in real time and to relocate their target 

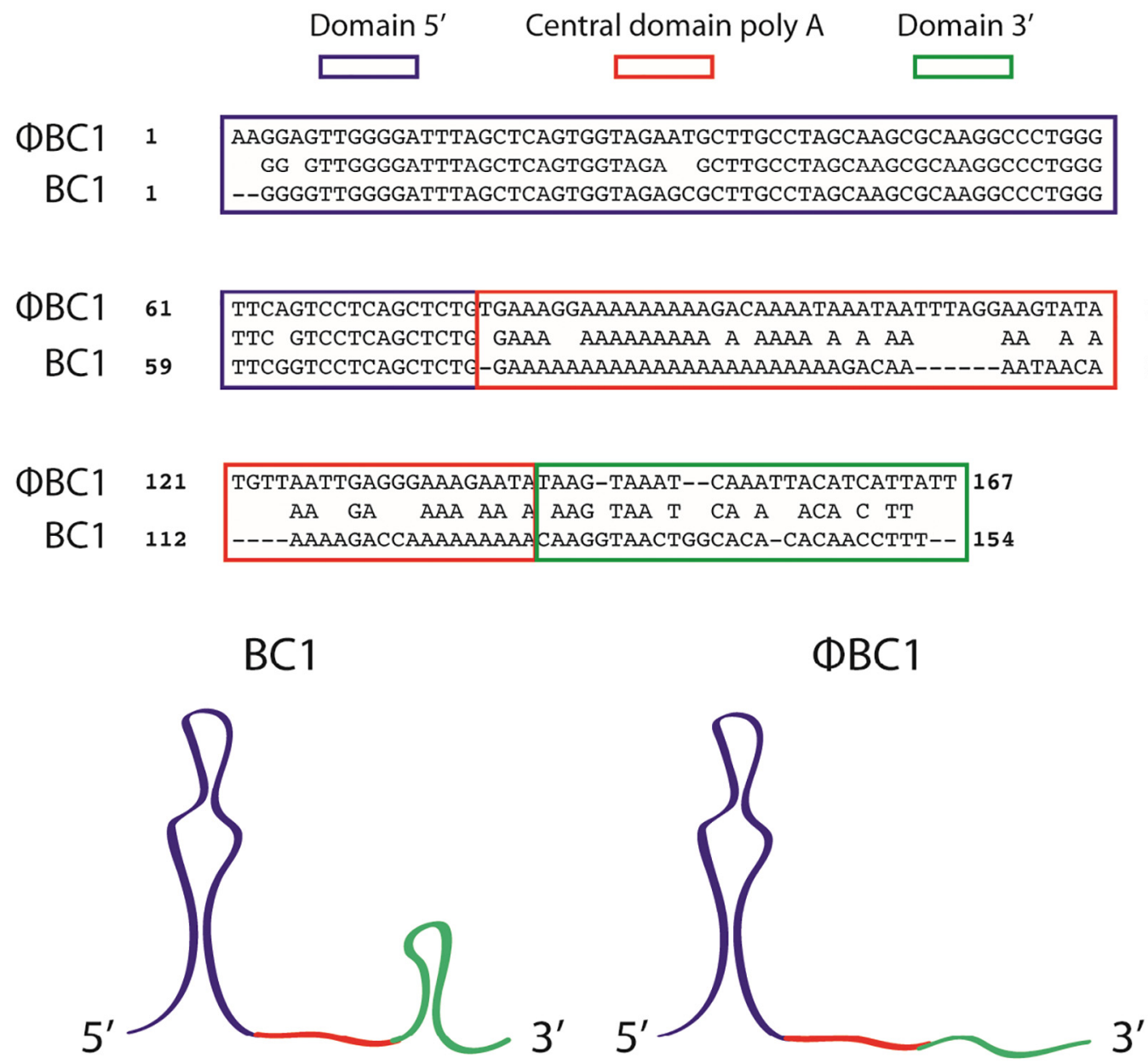

Fig. 1. Nucleotide sequence alignment of $B C 1$ (154-nt) and its pseudogene (ФBC1) (167-nt) and schematic representation of their secondary structure content. The $5^{\prime}$ domain is shown in blue, the central polyA domain is shown in red and the $3^{\prime}$ domain is shown in green. Overall, these sequences share an identity of $72 \%$. The represented secondary structure content of $B C 1$ has been determined experimentally, whereas the secondary structure elements of $\Phi B C 1$ have been predicted using the RNAfold server (http://rna.tbi.univie.ac.at/cgi-bin/RNAWebSuite/RNAfold.cgi) from the Vienna RNA Web services website (http://rna.tbi.univie.ac.at).

or to modulate its activity in cells and even in complete organisms [32-34].

In the standard procedure to generate nanobodies, the first step consists of immunizing a camelid with the targeted molecule. We expected that stRNAs will not be immunogenic, and therefore, they will not elicit an immune response of the heavy chain-only antibodies. Therefore, we describe herein the design, the construction and the phage display selection of a synthetic nanobody gene library dedicated for nucleic acid binding. The screening of this library led to the selection of, at least, one nanobody, named $c A b_{\mathrm{BC} 1 \mathrm{rib}} 3$, that binds to $\varphi B C 1$ with $\mathrm{nM}$ affinity. We also investigated the thermal denaturation of the stRNA in the presence or the absence of the selected nanobody. Our data showed that the protein does not influence the thermal denaturation and renaturation process. Finally, we demonstrated that although $\mathrm{CAb}_{\mathrm{BC} 1 \mathrm{rib}} 3$ is specific for stRNA, it recognizes different stRNAs, suggesting new strategies to improve the specificity of nanobodies.

\section{Results}

\section{Design and construction of a synthetic library of nanobodies dedicated for RNA binding}

We choose the nanobody, named cAb-BCll10, as the framework to create the synthetic library. It was initially selected from an immune library against Bcll $\beta$ lactamase. This nanobody binds to the $\beta$-lactamase active site and inhibits its enzymatic activity [35]. cAb$\mathrm{BCll10}$ is stable, tolerant to mutations and has been 
successfully used several times as a universal scaffold to exchange its antigen specificities by complementarity determining region (CDR) grafting $[36,37]$. In addition, its 3D structure has been solved and has revealed that its CDRs are well exposed on the protein surface for efficient binding. Finally, this nanobody is produced in Escherichia coli in high yields.

Based on different structural data available for nanobodies in complex with its antigen [36], 11 residues belonging to the antigen-binding regions (CDRs) were selected for randomization (3 residues within CDR1 and 8 residues within CDR3) (Fig. 2a). In the crystal structure of cAb-BCII10 (PDB ID: 3DWT), the side chains of these amino acids were all sticking out toward the solvent and are available for antigen interaction. Furthermore, this 3D structure also shows that these residues are clustered in the same area (Fig. 2c).

The gene library was synthetized using the "trinucleotide mutagenesis" (TRIM) technology (GeneArt $R$, Combinatorial DNA library), in which the 11 mutated residues were randomized in a way that it incorporates preferentially 6 residues that are most commonly found at the interfaces of protein/RNA complexes available in the PDB [38-40]. In practice, each randomized position was mutated using a subset of trinucleotide blocks encoding: $10 \%$ for Arg, $10 \%$ for Lys, $10 \%$ for Ser, $10 \%$ for Asn, $10 \%$ for Gly, $10 \%$ for Tyr and $40 \%$ for all other residues, except Cys, in equal amounts. The theoretical diversity of this library is estimated to be $\approx 19^{11}$. The non-amplified library effectively contained over $10^{11}$ independent molecules.
The library was amplified by PCR and ligated into the phagemid vector pHEN4 [41]. The ligation product was then transformed into TG1 cells. The obtained library comprised $\approx 10^{10}$ individual transformants of which $\approx 80 \%$ harbored a pHEN4 vector with an insert compatible with the size of a cAb-BCll10 variant. To assess the quality of the library, the gene of 100 randomly chosen clones was sequenced in order to determine the percentage of amino acid incorporation for each randomized position. The amino acid distribution in the randomized loops as obtained from DNA sequencing is presented in Fig. 3 and confirms an amino acid occurrence that correlates well with our design.

\section{Selection of stRNA-binding nanobodies}

The nanobodies of the dedicated RNA-binding nanobody library were displayed at the tip of $\mathrm{M} 13$ bacteriophages and fused to the coat protein PIII, and consecutive rounds of selection were performed against the target stRNA generated by in vitro transcription.

Importantly, since the function of $\varphi B C 1$ is unknown, we had no mean to assess the folding of this stRNA into its native conformation after in vitro transcription. This is why we decided to fuse $\varphi B C 1$ to a catalytic stRNA, named rib ${ }_{g l m s}$, as follows: ${ }^{5^{\prime}} \varphi B C 1-$ rib $_{g l m s}^{3^{\prime}}$. This $172-\mathrm{nt}-$ long ribozyme, is expressed by Bacillus anthracis and originates from the $5^{\prime}$ untranslated region of the glucosamine-6-phosphate (Glc6P) synthase messenger RNA [42]. In the presence of Glc6P, rib glms $_{\text {auto- }}$ cleaves itself from the mRNA from which it takes part.

(a)

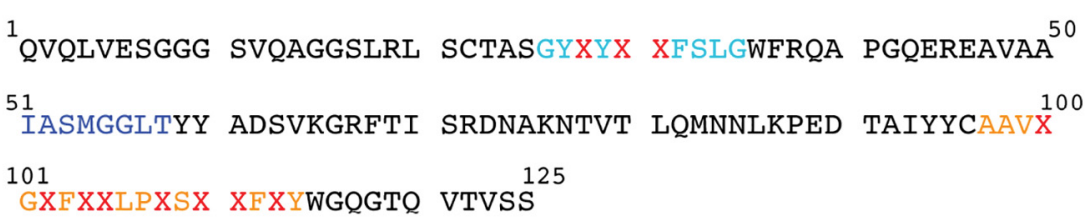

(b)

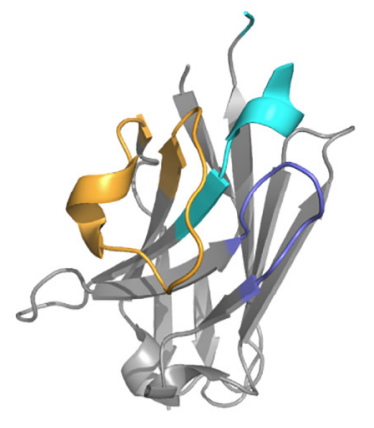

(c)

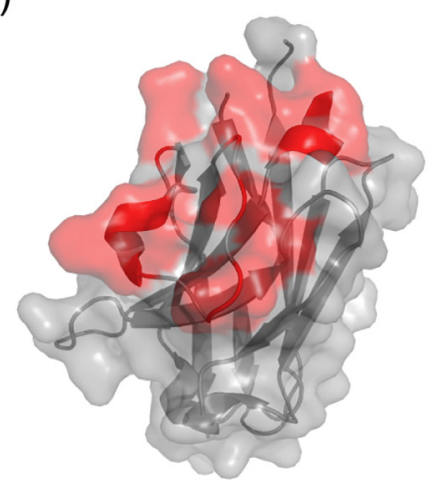

Fig. 2. Representation of the amino acid sequence (a) and $3 \mathrm{D}$ structure of cAbBCIl-10 (PDB ID: 3DWT) (b and $c)$. The residues that belong to the CDRs are represented in cyan, blue and orange for CDR-1, -2 and -3 , respectively, in the amino acid sequence (a) and on the 3D structure (b). The randomized residues are highlighted in red in the amino acid sequence $(A)$ as well as on nanobody structure surface shown in panel (c). 


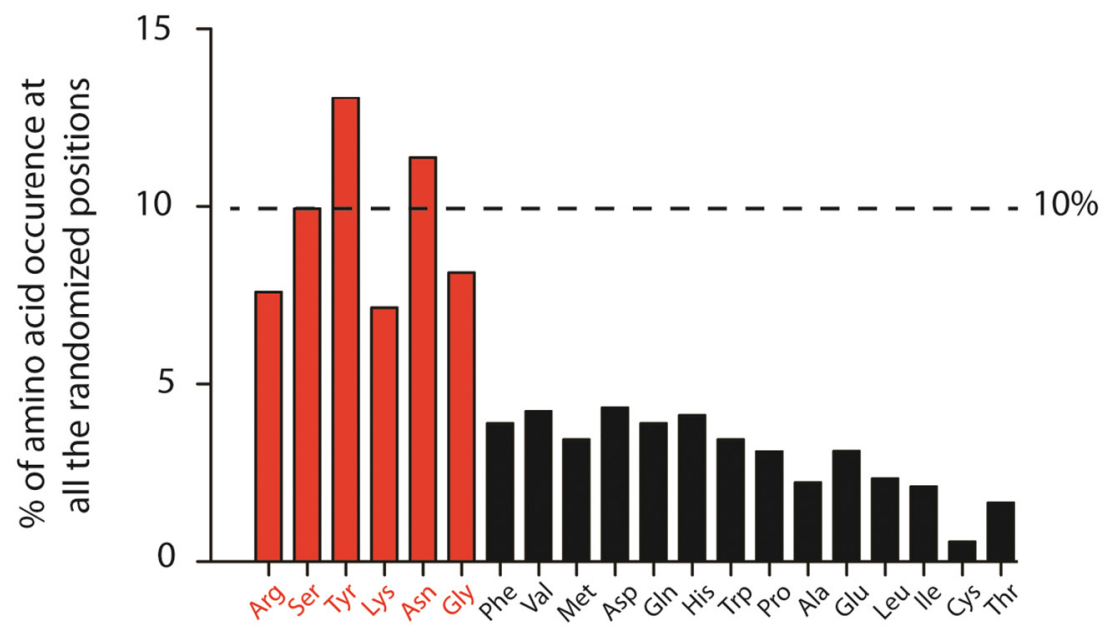

Fig. 3. Representation of the amino acids distribution at the randomized positions. The bars represent the occurrence of a particular amino acid (in \%) across all the randomized positions. The residues that were preferentially introduced during the mutagenesis are represented in red and should theoretically be $10 \%$ (dashed line). The percentage of amino acid occurrence was calculated from the sequences of 100 cherry picked clones from the initial library.

This catalytic activity has been well characterized [43] and can be easily measured (see Materials and Methods). Therefore the rib $\mathrm{glms}_{\mathrm{m}}$ activity was monitored after each transcription as a quality control experiment. Although, the presence of the ribozyme activity does not reflect the conformation of the $\varphi B C 1$ moiety of the fusion RNA, it indicates that the in vitro transcription conditions were compatible with the native folding an stRNA.

In total, we performed five rounds of selection against the fusion stRNA: ${ }^{{ }^{\prime}} \varphi B C 1-\mathrm{rib}_{\mathrm{g} / m s}^{3^{\prime}}$, after checking the presence of the ribozyme activity (data not shown). At the end of the selection process, 95 individual clones from rounds 3, 4 and 5 were cultured and recombinant proteins expressed in the periplasmic space of $E$. coli in fusion with a hemagglutinin $(\mathrm{HA})$ tag at their $\mathrm{C}$-terminal end. The periplasmic proteins were extracted and used in an ELISA to identify the variants that bind to the target RNA (data not shown). Only three of the tested clones $\left(c A b_{B C 1 r i b} 3, c A b_{B C 1 r i b} 20, c A b_{B C 1 \text { rib }} 90\right)$ gave positive signals in ELISA. The nanobodies $\mathrm{CAb}_{\mathrm{BC} \text { rib } 3}$ and $C A b_{B C 1 r i b} 90$ were selected from the third round of biopanning, and $\mathrm{CAb}_{\mathrm{BC} 1 \mathrm{rib}} 20$ was retrieved after the fifth round of bio-panning. Bio-layer interferometry (BLI) measurements were performed to confirm the ELISA results. The data obtained show that significant binding against immobilized $\varphi B C 1-$ rib ${ }_{\text {glms }}$ could only be detected for $\mathrm{CAb}_{\mathrm{BC} 1 \mathrm{rib}} 3$ and $\mathrm{CAb}_{\mathrm{BC} 1 \text { rib }} 90$ (Fig. 4a). These two nanobodies were therefore sub-cloned into the pHEN6 expression vector for further characterization. Remarkably, the sequence of $\mathrm{CAb}_{\mathrm{BC} 1 \text { rib }} 90$ contains a single substitution in a non-randomized position of its CDR3 (A97V) (Fig. 4b). Given that the phage display screening led to the retrieval of this variant, we decided to keep this spontaneous mutation.

After sub-cloning the nanobody genes in pHEN6, only $c A b_{B C 1 r i b} 3$ could be expressed in significant amount. We therefore focused our study on $c A b_{B C 1 \text { rib }} 3$. When expressed from pHEN6, it harbors a C-terminal $\mathrm{His}_{6}$ tag to facilitate its purification by nickel ion affinity chromatography. The protein sample was then further purified to homogeneity by cationic exchange chromatography.

\section{Characterization of the interaction between $\mathrm{CAb}_{\mathrm{BC} 1 \mathrm{rib}} 3$ and $\varphi B C 1-$ rib $_{\mathrm{glms}}$}

\section{Determination of the binding specificity of $c A b_{B C 1 r i b} 3$}

First, we analyzed the association of $c A b_{B C 1 r i b} 3$ with different control molecules to assess its specificity for stRNAs. Besides the $\varphi B C 1-$ rib $_{\text {glms }}$ fusion, the other target molecules include the following: ssDNA

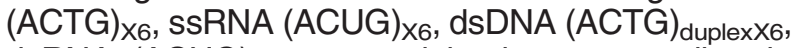
dsRNA (ACUG) duplex $\times 6$ and bovine serum albumin (BSA), a negatively charged protein. Apart from the strong $\varphi B C 1$-rib ${ }_{\text {glms }}$ recognition, a very weak binding was observed for the double stranded RNA molecule (Fig. 5), whereas all the other tested molecules were not recognized at all. This result strongly suggests that $c A b_{\mathrm{BC} 1 \text { rib }} 3$ is specific for stRNA.

Qualitative binding assays were then performed in order to identify the exact partner of the $\varphi B C 1-$ rib $_{g / m s}$ RNA fusion (i.e., $\varphi B C 1$ or rib ${ }_{g l m s}$ ) that is recognized by the nanobody. The data presented in Fig. $6 a$ show that $\mathrm{CAb}_{\mathrm{BC} 1 \mathrm{rib}} 3$ recognizes the $\varphi B C 1$ part of the fused RNA since hardly any binding could be detected for the isolated $\mathrm{rib}_{\mathrm{glms}}$. Moreover, to further investigate the discrimination ability of $\mathrm{CAb}_{\mathrm{BC} 1 \mathrm{rib}} 3$, BLI measurements were performed against different stRNAs not related to $\varphi B C 1$ or rib ${ }_{g / m s}$ in terms of sequences and predicted functions (see Materials and Methods). The stRNA ctrl 1 is 7SK, a human 331-nt-long small nuclear ncRNA that acts as a scaffold in the nuclear ribonucleoprotein complex. This complex was shown to play a key role in regulating the activity of the positive transcription elongation factor $b$ (P-TEFb) [44]. The stRNA ctrl 2 is a bacterial 159-nt-long domain (the P4-P6 domain) of the group I intron ribozyme expressed Tetrahymena thermophyla [45]. Our data indicated that both control stRNAs tested are recognized by $\mathrm{cAb}_{\mathrm{BC} 1 \text { rib }} 3$, however, the nanobody exhibits differential binding profiles to 
(a)

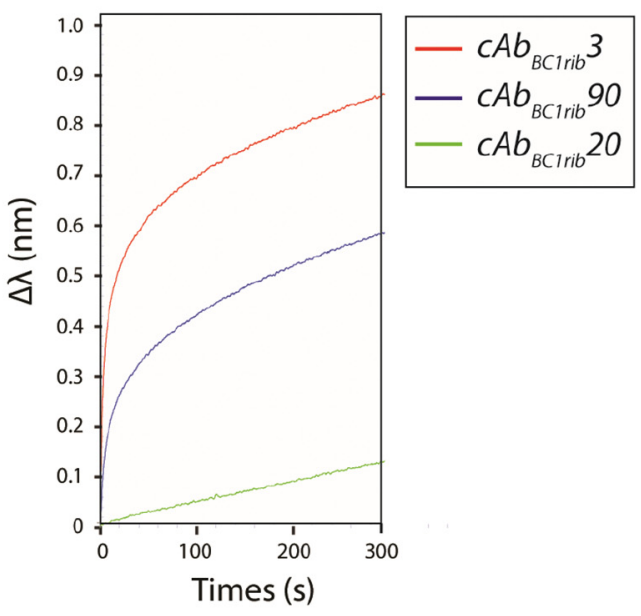

(b)

$\begin{array}{lll}\text { CDR1 CDR2 } & \text { CDR3 }\end{array}$
$\mathrm{CAb}_{\text {BC1rib }} 3$ GYGYRNFSLG IASMGGLT AAVRGRFYPLPRSYNFGY
$\mathrm{CAb}_{\text {BC1rib }} 90$ GYVYPRFSLG IASMGGLT VAVSGKFYKLPYSPNFWY
$C A b_{\text {BC1rib }} 20$ GYPYEMFSLG IASMGGLT AAVTGRFOGLPFSKVFRY

Fig. 4. Binding measurements of the selected nanobodies with the fusion RNA $\varphi B C 1$-rib $_{\text {glms }}$ performed by BLI (a). The experiment was performed using an "anti-mouse" sensor on which an anti-HA antibody was immobilized. Then periplasmic extracts containing nanobodies were loaded onto the sensor via their Cterminal $\mathrm{HA}$ tag. The analyte $(\varphi B C 1-$ rib $_{\text {glms }}$ ) concentration used for these assays was $100 \mathrm{nM}$. The association was monitored for $300 \mathrm{~s}$. CDR amino acid sequence alignment of $\mathrm{cAb}$ $\mathrm{BC}$ rib3, $\mathrm{CA} \mathrm{b}_{\mathrm{BC} \text { rib }} 90$ and $\mathrm{CAb}_{\mathrm{BC} 1 \mathrm{rib}} 20$ (b). The randomized residues are indicated in red; the A97V mutation present in the $\mathrm{CDR} 3$ for $\mathrm{CAb}_{\mathrm{BC} 1 \mathrm{rib}} 3$ is underlined.

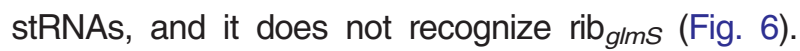
These observations suggest that it probably recognizes a common epitope shared by $\varphi B C 1$ and the 2 control stRNAs.

In order to further investigate the differential binding of $c A b_{\mathrm{BC} 1 \text { rib }} 3$ among stRNA targets, qualitative binding experiments were conducted on $B C 1$ and $\varphi B C 1$ and are compared in Fig. 7. As mentioned in the introduction, these two stRNAs mostly differ from each other in the central polyA and $3^{\prime}$ domains. Our results demonstrate that the observed association rates reach saturation at a lower concentration for $\varphi B C 1$ compared to $B C 1$, whereas, as observed above, the dissociation rates are very slow and highly similar for both stRNAs and can be considered as negligible. These data suggest that the binding of $c A b_{\mathrm{BC} 1 \mathrm{rib}} 3$ to $B C 1$ is weaker compared to that of $\varphi B C 1$. In order to further compare the binding efficiencies of $c A b_{B C 1 \text { rib }} 3$ to these stRNAs, observed initial rates of association were plotted against the RNA concentration and fitted using a hyperbola equation (Fig. 7c). The fits predict at least a four-fold higher binding efficiency for $\varphi B C 1$ compared to $B C 1$. This result is important as it provides evidence that $c A b_{B C 1 \text { rib }} 3$ presents a differential recognition between $B C 1$ and $\varphi B C 1$ and across stRNAs, although the origin for this differential binding behavior remains unclear and will be discussed later (see Discussion). Notably, additional BLI control experi-

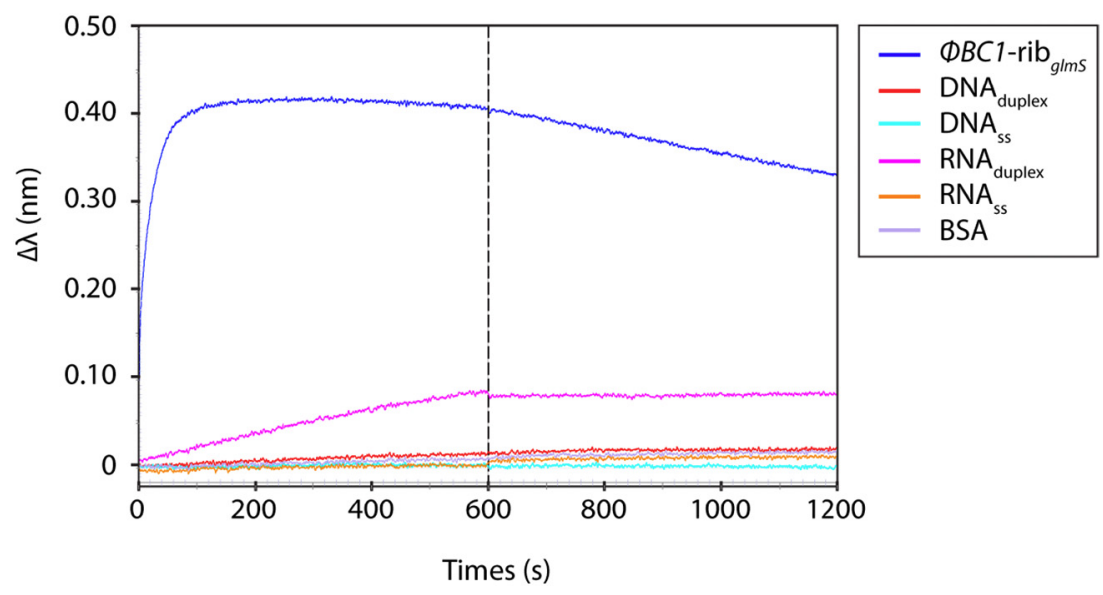

Fig. 5. Qualitative binding of

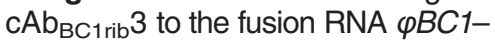
$\mathrm{rib}_{g / m s}$ and the different control molecules measured by BLI. The experiment was performed using an "anti-His" sensor on which purified $\mathrm{CAb}_{\mathrm{BC} 1 \mathrm{rib}} 3$ was immobilized via its C-terminal $\mathrm{His}_{\mathrm{x} 6}$ tag. The concentration of the different analytes used for these assays was $100 \mathrm{nM}$. The association and dissociation phases were both measured for $600 \mathrm{~s}$. 
(a)

a)

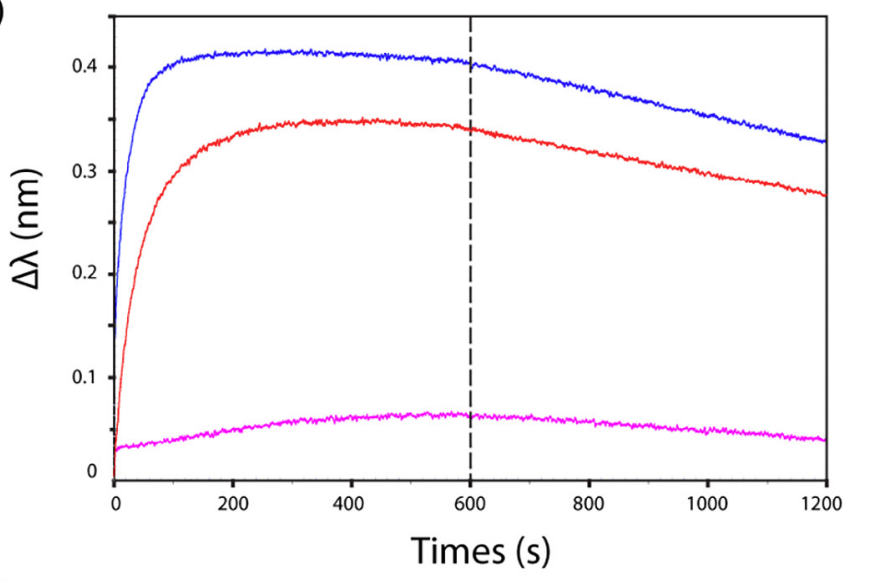

$\begin{array}{ll}- & \Phi B C 1-\mathrm{rib} \\ - & \Phi B C 1 \\ - & \text { rib } \\ & \end{array}$

(b)
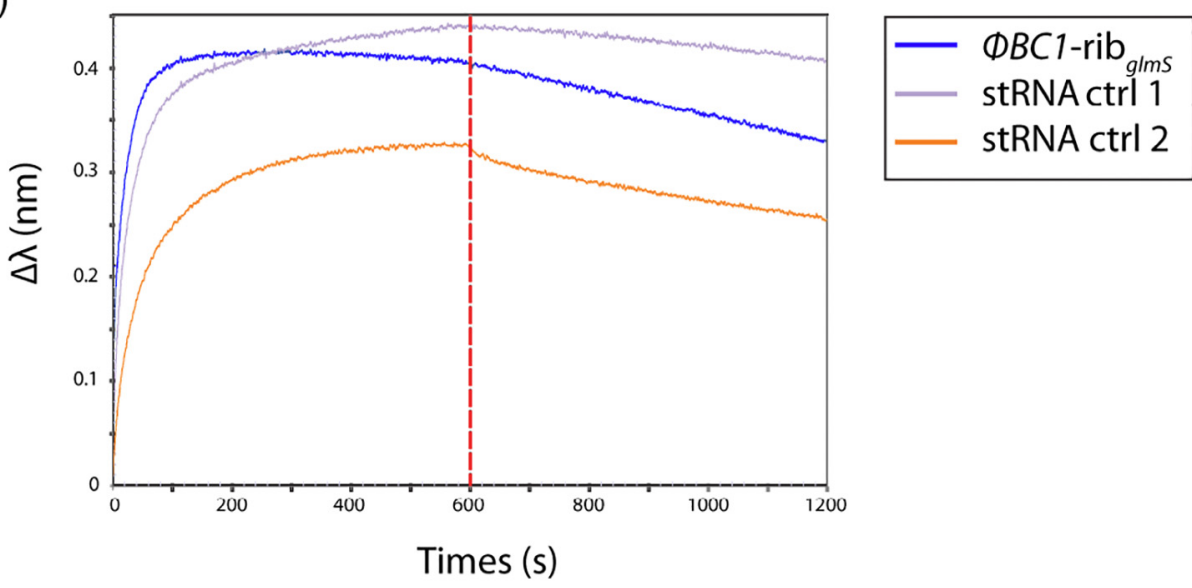

Fig. 6. Qualitative binding measurements of $c A b_{B C 1 r i b} 3$ to different stRNAs performed by BLI. (a) Binding curves of the

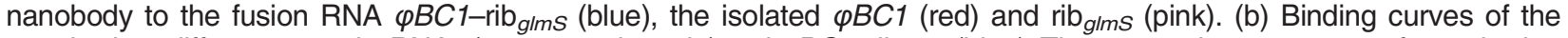
nanobody to different control stRNAs (orange and purple) and $\varphi B C 1$-rib ${ }_{g l m s}$ (blue). These experiments were performed using an "anti-His" sensor on which purified $\mathrm{CAb}_{\mathrm{BC} 1 \mathrm{rib}} 3$ was immobilized via its $\mathrm{C}$-terminal His $\mathrm{x}_{\mathrm{6}}$ tag. The analytes (stRNAs) concentration used for these assays was $100 \mathrm{nM}$, and the association and dissociation phases were both measured for $600 \mathrm{~s}$.

ments were performed in order to assess potential nonspecific binding behaviors of both stRNAs. These data are presented in the Supplemental Data (Fig. S1). Briefly, a control nanobody was immobilized on BLI sensors and binding assays with $\varphi B C 1$ and $B C 1$ were conducted to assess potential differences in nonspecific binding of these two stRNAs. The results indicate that only a very weak binding could be observed in the presence of an excess $(1 \mu \mathrm{M})$ of $B C 1$ but not in the presence of $100 \mathrm{nM}$. In the case of $\varphi B C 1$, no binding could be detected in the presence of either $100 \mathrm{nM}$ or $1 \mu \mathrm{M}$. These data suggest that, at high concentrations, $\mathrm{BC} 1$ has higher tendency to exhibit non-specific binding compared to $\varphi B C 1$.

\section{Determination of the binding affinity between $c A b_{B C 1 r i b} 3$ and $\varphi B C 1-r i b_{g l m s}$}

Quantitative BLI measurements were carried out to determine the binding affinity of $\mathrm{CAb}_{\mathrm{BC} 1 \text { rib }} 3$ for $\varphi B C 1$ alone or in the context of the RNA fusion. The binding assays (Fig. 8) show that $\mathrm{CAb}_{\mathrm{BC} 1 \mathrm{rib}} 3$ binds to $\varphi B C 1-$ rib $_{g l m s}$ and to $\varphi B C 1$ with $\mathrm{nM}$ affinity $\left(K_{\mathrm{D}}=2.3 \pm\right.$ $0.8 \times 10^{-9} \mathrm{M}$ and $K_{\mathrm{D}}=6.8 \pm 2.8 \times 10^{-9} \mathrm{M}$, respectively). The experimental data were adjusted using a 1:1 mathematical binding model. The equilibrium dissociation constants and the kinetic parameters $\left(k_{\text {on }}\right.$ and $\left.k_{\text {off }}\right)$ presented in Table 1 are very similar to those measured for nanobodies directed against proteins selected from immune libraries [41].

As explained in the introduction section, it is well established that the 3D structure of folded RNAs is stabilized by the presence of bivalent ions such as $\mathrm{Mg}^{2+}$. Therefore, we have measured the binding affinities of $\mathrm{CAb}_{\mathrm{BC} 1 \mathrm{rib}} 3$ for the fusion stRNA and the isolated $\varphi B C 1$ in the presence of a chelating agent (10 mM EDTA) to assess whether the recognized epitope is a tertiary structure element or a secondary structure element. Circular dichroism (CD) spectra of the $\varphi B C 1-$ rib $_{g l m s}$ recorded in the presence of EDTA 
(a)

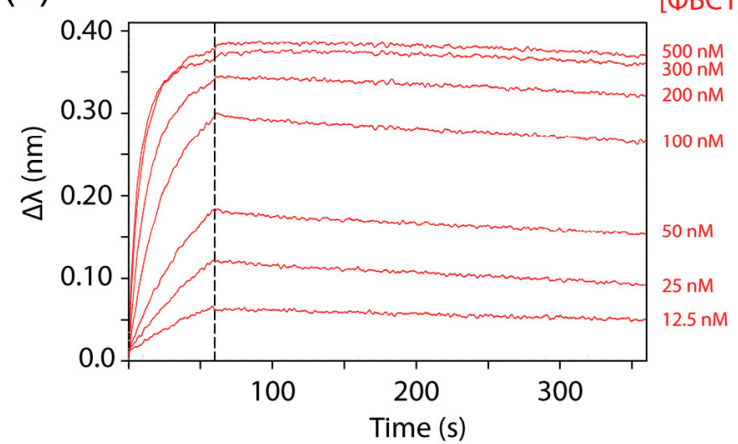

(c)

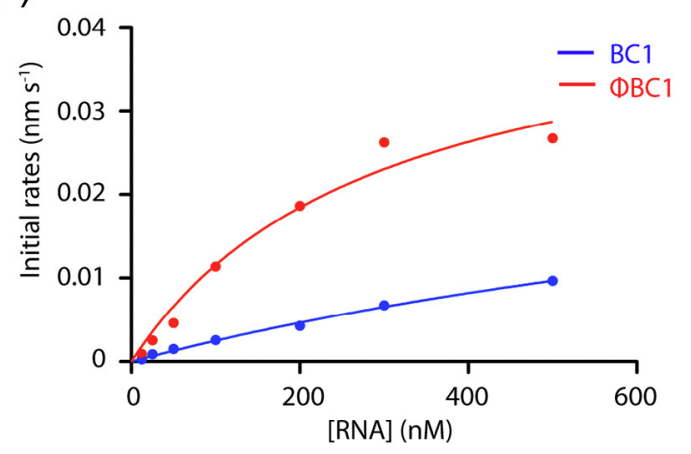

(b)

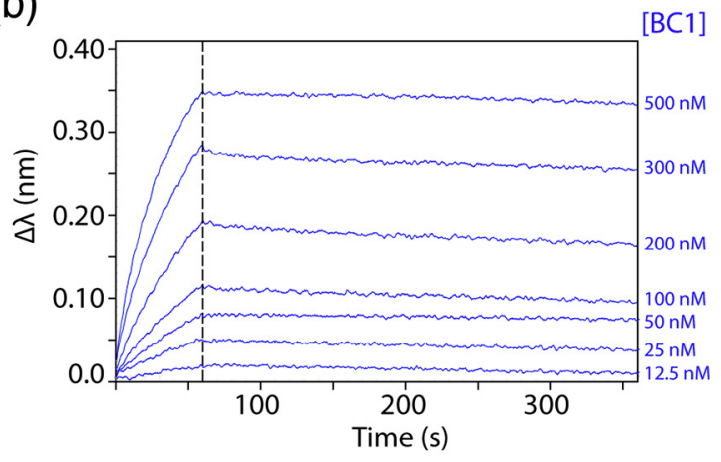

Fig. 7. Binding measurements of $\mathrm{CAb}_{\mathrm{BC} \text { 1rib }} 3$ to $\Phi B C 1$ (red) (a) and $B C 1$ (blue) (b) performed by BLI. These experiments were performed using an "anti-His" sensor on which $\mathrm{cAb}_{\mathrm{BC} 1 \mathrm{rib}} 3$ was loaded and immobilized via its $\mathrm{C}$-terminal His $\mathrm{x}_{6}$ tag. The stRNAs concentrations used for these assays were 12.5, 25, 50, 100, 200, 300 and $500 \mathrm{nM}$. The association and dissociation phases were measured for 60 and $300 \mathrm{~s}$, respectively. The measured rates of association (initial rates) were plotted against the stRNA concentrations (c) and fitted to a hyperbola equation in order to compare the binding efficiencies of $\mathrm{CAb}_{\mathrm{BC} 1 \mathrm{rib}} 3$ to $\Phi B C 1$ and $B C 1$.

(data not shown) indicated that the stRNAs conserves its secondary structure content. The $K_{\mathrm{D}}$ values decrease 100-fold for both $\varphi B C 1$-rib ${ }_{g l m s}$ and $\varphi B C 1$ alone in the presence of EDTA (i.e., in the absence of $\mathrm{Mg}^{2+}$ ) (Fig. 9). The kinetic parameters (Table 1) indicate that, for both RNAs, this strong increase in the equilibrium binding affinity (decrease of the dissociation constant) in the presence of EDTA is mostly due to an increase of the $k_{\text {on }}$ (10- to 40-fold) combined to with a 10- to 4-fold decrease of the $k_{\text {off }}$. Interestingly, qualitative binding assays conducted by $\mathrm{BLI}$ on the control stRNAs presented similar behaviors in the presence of EDTA, namely faster association and slower dissociation rates (data not shown). Additional control experiments were performed in the presence of EDTA in order to assess the non-specific binding of the stRNA on the BLI surface. These experiments are presented in the Supplemental Data (Fig. S2). Briefly, in the absence of $\mathrm{Mg}^{2+}$, no binding could be detected between rib $_{g l m s}$ (a stRNA not recognized by $\left.c A b_{B C 1 r i b} 3\right)$ and $B L I$ sensors on which a control nanobody was immobilized, suggesting that the stRNA does not stick on the BLI surface when its 3D structure is abolished. In contrast, significant signals could be detected between rib glms $_{\text {and }} \mathrm{cAb}$ $\mathrm{BC} 1$ rib3 loaded on $\mathrm{BLI}$ sensors in the absence of $\mathrm{Mg}^{2+}$, whereas in the presence of $\mathrm{Mg}^{2+}$, no binding was observed (Fig. 6a). However, the signals measured in the absence of $\mathrm{Mg}^{2+}$ are not dose dependent and present atypical shapes, suggesting that these signals probably reflect non-specific binding of rib ${ }_{g / m s}$ on $\mathrm{CAb}_{\mathrm{BC} 1 \mathrm{rib}} 3$ only observed in the absence of $\mathrm{Mg}^{2+}$.

All together, these data suggest that $\mathrm{CAb}_{\mathrm{BC} 1 \mathrm{rib}} 3$ probably recognizes a secondary structure element present in $\varphi B C 1$ and in the different control stRNAs tested. This observation will be further developed in the Discussion section.

\section{Effects of the interaction of $c A b_{B C 1 \text { rib }} 3$ on the properties of $\varphi B C 1-$ rib $_{\mathrm{glms}}$}

In order to investigate the potential effects of the binding of the nanobody on the structure and stability of the stRNA, we recorded CD spectra at $25{ }^{\circ} \mathrm{C}$ and we monitored the changes of CD signal at $223 \mathrm{~nm}$ or $267 \mathrm{~nm}$ as a function of the temperature for the purified nanobody, the stRNA and for the nanobody: stRNA complex. 
(a)

(b)

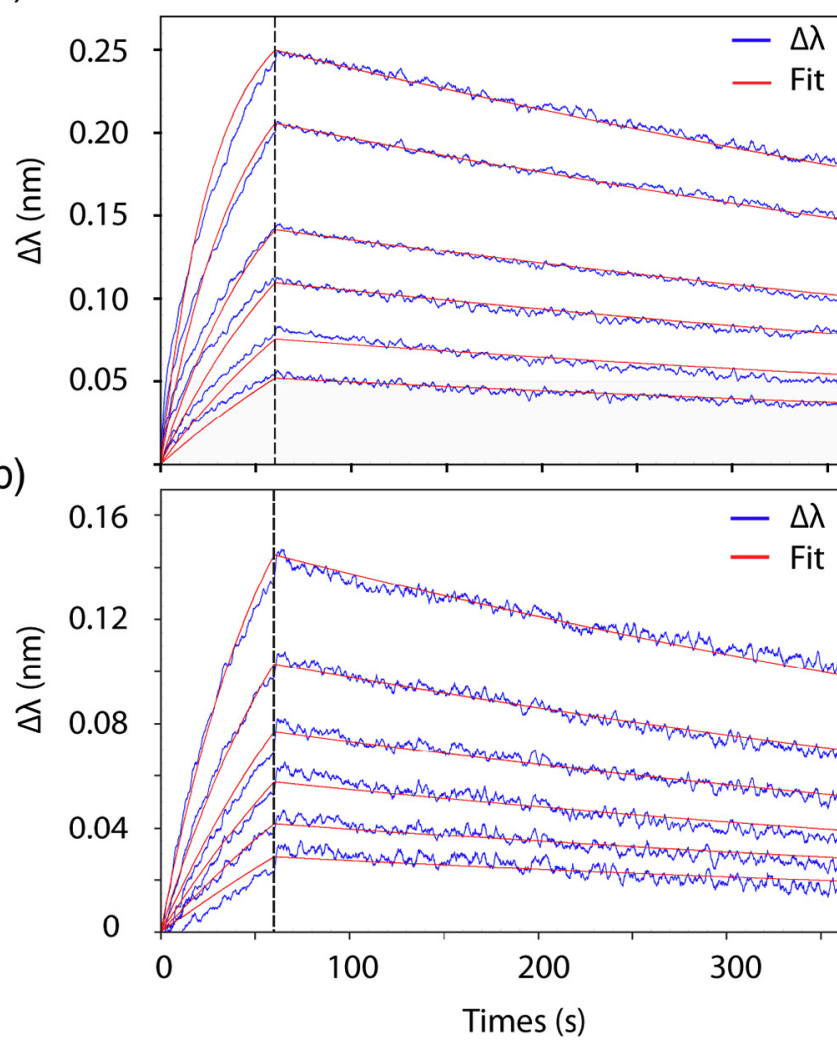

\author{
$\left[\varnothing B C 1-\right.$ rib $\left._{g / m s}\right]$ \\ $100 \mathrm{nM}$ \\ $66.7 \mathrm{nM}$ \\ $44.4 \mathrm{nM}$ \\ $29.6 \mathrm{nM}$ \\ $19.7 \mathrm{nM}$ \\ $13.1 \mathrm{nM}$
}

$[\varnothing B C 1]$

Fig. 8. Quantitative binding measurements of $c A b_{B C 1 \text { rib }} 3$ to the fusion RNA $\varphi B C 1-$ rib $_{g / m s}$ (a) and the isolated $\varphi B C 1$ (b) performed by $\mathrm{BLI}$ in the presence of $10 \mathrm{mM}$ $\mathrm{MgCl}_{2}$. These experiments were performed using an "anti-His" sensor on which purified $\mathrm{CAb}_{\mathrm{BC} 1 \mathrm{rib}} 3$ was loaded and immobilized via its $\mathrm{C}$ terminal $\mathrm{His}_{\mathrm{x} 6}$ tag. The analyte (stRNAs) concentration used for these assays are indicated on the graphs. The association and dissociation phases were monitored for 60 and $300 \mathrm{~s}$, respectively. The experimental data were fitted using a 1:1 binding model.

Effects of the binding of the nanobody on the structure of $\varphi B C 1-r i b_{g l m s}$

Figure 10 represents the CD spectra for $\varphi B C 1-$ $\mathrm{rib}_{\text {glms }}$ and $\mathrm{CAb}_{\mathrm{BC} 1 \mathrm{rib}} 3$ alone or in complex at a 1:1 molar ratio cAb:stRNA. The spectrum recorded for $\varphi B C 1-$ rib $_{g l m s}$ indicates that the stRNA possesses different secondary structure elements. The positive and intense signal observed around $267 \mathrm{~nm}$ is indicative for the presence of right-handed helices contained in hairpins, whereas the negative signal less intense around $210 \mathrm{~nm}$ is attributed to the presence of parallel duplexes [46]. The spectrum measured for the nanobody alone presents typical features of nanobodies, with a positive signal observed around $223 \mathrm{~nm}$ that indicates the presence of aromatic residues immobilized within a particular chemical environment suggesting the presence of a well-defined 3D structure [47]. The second positive signal recorded at $203 \mathrm{~nm}$ is due to peptide backbone, which, in this case, is an antiparallel $\beta$-sheet [47]. It is interesting to see that the spectra of the complex are very similar to the one measured for the stRNA alone, suggesting that the binding of the nanobody does not alter the secondary structure content of the stRNA.

\section{Effects of the binding on the thermal unfolding of $\varphi B C 1-r i b_{g l m s}$}

The thermal denaturation of $\mathrm{CAb}_{\mathrm{BC} 1 \mathrm{rib}} 3$ alone, $\varphi B C 1-$ rib $_{\text {glms }}$ alone or in complex with $\mathrm{cAb}_{\mathrm{BC} \text { 1rib }} 3$

Table 1. Equilibrium and kinetic constants values measured for the interaction between $\mathrm{cAb}_{\mathrm{BC} 1 \mathrm{rib}} 3$ and $\varphi B C 1$ in the context of the fusion RNA or isolated

\begin{tabular}{lcccc}
\hline & EDTA & $k_{\text {on }}\left(\mathrm{M}^{-1} \mathrm{~s}^{-1}\right)$ & $k_{\text {off }}\left(\mathrm{s}^{-1}\right)$ & $K_{\mathrm{D}}(\mathrm{M})$ \\
\hline$\varphi B C 1-$ rib $_{\text {glms }}$ & - & $2.9 \pm 2.5 \times 10^{5}$ & $1.7 \pm 1.0 \times 10^{-3}$ & $2.3 \pm 0.8 \times 10^{-9}$ \\
$\varphi B C 1-\mathrm{rib}_{\text {glms }}$ & + & $3.5 \pm 1.2 \times 10^{6}$ & $1.1 \pm 1.4 \times 10^{-4}$ & $2.5 \pm 3.3 \times 10^{-11}$ \\
$\varphi B C 1$ & - & $3.3 \pm 1.7 \times 10^{5}$ & $2.1 \pm 0.9 \times 10^{-3}$ & $6.8 \pm 2.8 \times 10^{-9}$ \\
$\varphi B C 1$ & + & $1.2 \pm 0.2 \times 10^{7}$ & $8.2 \pm 4.8 \times 10^{-3}$ & $7.2 \pm 4.8 \times 10^{-11}$ \\
\hline
\end{tabular}

The association and dissociation kinetic constants $\left(k_{\mathrm{on}}\right.$ and $\left.k_{\mathrm{off}}\right)$ and the deduced equilibrium dissociation constants $\left(K_{\mathrm{D}}\right)$ were measured by BLI. The experimental data were fitted using a 1:1 model using at least six analyte (stRNA) concentrations. The $K_{\mathrm{D}}$ values and associated errors correspond to averages and standard deviations calculated from at least three independent BLI experiments. 
(a)

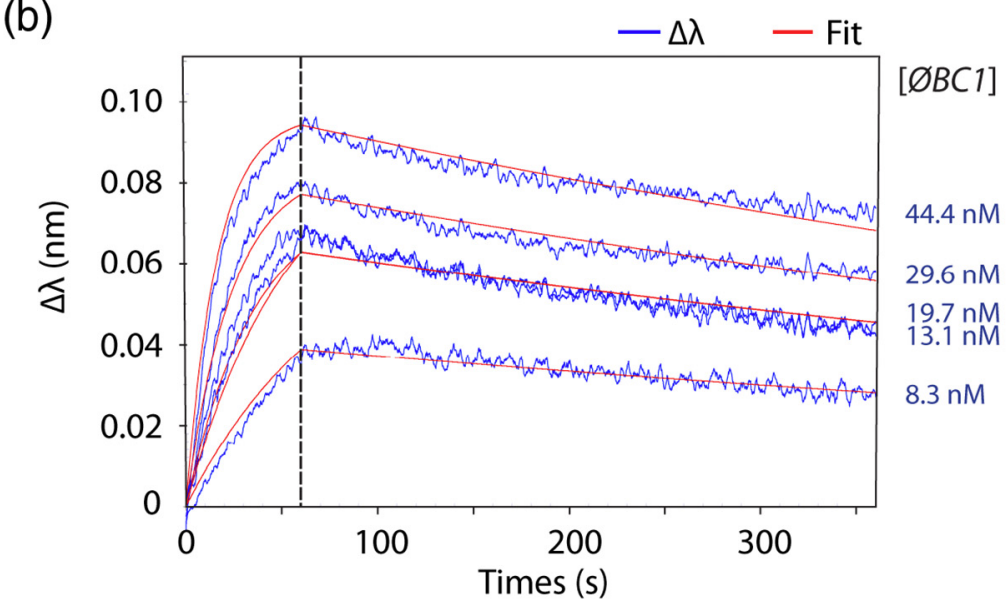

Fig. 9. Quantitative binding measurements of $c A b_{B C 1 \text { rib }} 3$ to the fusion RNA $\varphi B C 1-$ rib $_{g l m s}$ (a) and the isolated $\varphi B C 1$ (b) performed by $\mathrm{BLI}$ in the presence of $10 \mathrm{mM}$ EDTA. These experiments were performed using an "anti-His" sensor on which purified $\mathrm{CAb}_{\mathrm{BC} 1 \text { rib3 }} 3$ was immobilized via its C-terminal $\mathrm{His}_{\mathrm{x} 6}$ tag. The analyte (stRNAs) concentrations used for these assays are indicated on the graphs. The association and dissociation phases were monitored for 60 and $300 \mathrm{~s}$, respectively. The experimental data were fitted using a 1:1 binding model.
(1:1 molar ratio) was monitored by far UV CD at $223 \mathrm{~nm}$ for $\mathrm{cAb}_{\mathrm{BC} 1 \mathrm{rib}} 3$ and at $267 \mathrm{~nm}$ for the stRNA alone or in the complex. Note that at $267 \mathrm{~nm}$, there is no signal from the nanobody, then the unfolding of the stRNA within the complex can be monitored specifically. The results shown in Fig. $11 \mathrm{a}$ indicate that the denaturation and renaturation of $\mathrm{CAb}_{\mathrm{BC} 1 \text { rib } 3 \text { are coop- }}$ erative and characterized by an apparent two-state system. Noticeably, $c A b_{\mathrm{BC} \text { rib }} 3$ is thermostable since its temperatures of mid-denaturation upon heating $(\mathrm{Tm})$ is $68.4 \pm 0.4{ }^{\circ} \mathrm{C}$ (Table 2). Most of the $\mathrm{CD}$ signal is recovered after the renaturation process, indicating that the thermal denaturation is highly reversible as regularly observed for nanobodies [45].

The denaturation/renaturation process of $\varphi B C 1-$ rib $_{\text {glms }}$ exhibits distinct features compared to that of the nanobody. First, the denaturation is a lot less cooperative and occurs on an extended range of temperatures (from $\approx 25$ to $90^{\circ} \mathrm{C}$ ). A plausible explanation is that the different secondary structure elements of the stRNA act as sub-domains that have different thermal stabilities and therefore explains this non-cooperative behavior. Another explanation would be that the RNA unfolding occurs similarly to a "zipper" in analogy to the complementary strands of RNA duplexes and hairpins that separate from each other. Since the far UV CD measurements monitor only changes in the secondary structure of RNA and not its tertiary structure, it is not possible to determine if the denaturation of the two types of structures is cooperative. The temperature of mid denaturation of the secondary structure $(\mathrm{Tm})$ obtained for the denaturation curve is $70.7 \pm 2.2^{\circ} \mathrm{C}$. Interestingly, the renaturation process occurs at much lower temperature (Tm: $62.0 \pm 4.0^{\circ} \mathrm{C}$ ) compared to the denaturation (Fig. 11b and Table 2). This discrepancy between the unfolding and refolding transitions, known as hysteresis, has been described for nucleic acids [48]. Briefly, during the renaturation process, the probability of exact alignments of base sequences to reconstitute hairpins, duplexes and bulges present in the native stRNA conformation is very small. This means that non-native duplexes may form nucleation complexes that cannot be extended, resulting in large numbers of nonproductive intermediate states. These nucleated regions form and disassociate as the system seeks the lowest freeenergy (native) state. This phenomenon explains the observed hysteresis $[49,50]$.

Notably, the binding of $c A b_{B C 1 r i b} 3$ to the stRNA does not affect its denaturation or renaturation 
(a)

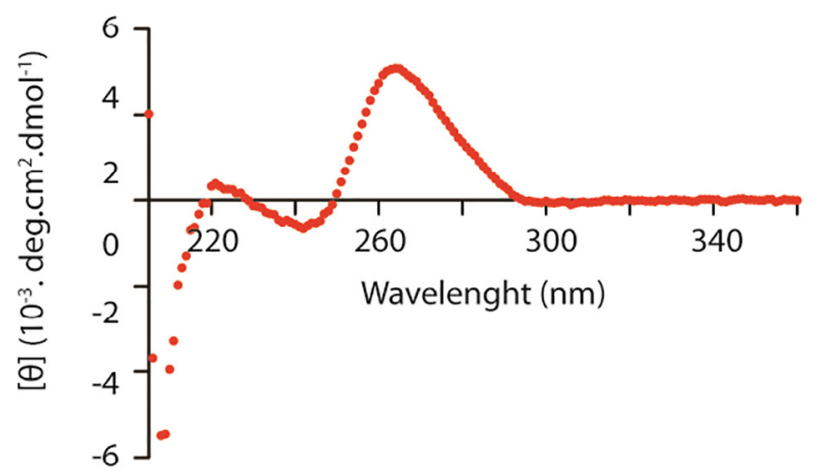

(b)

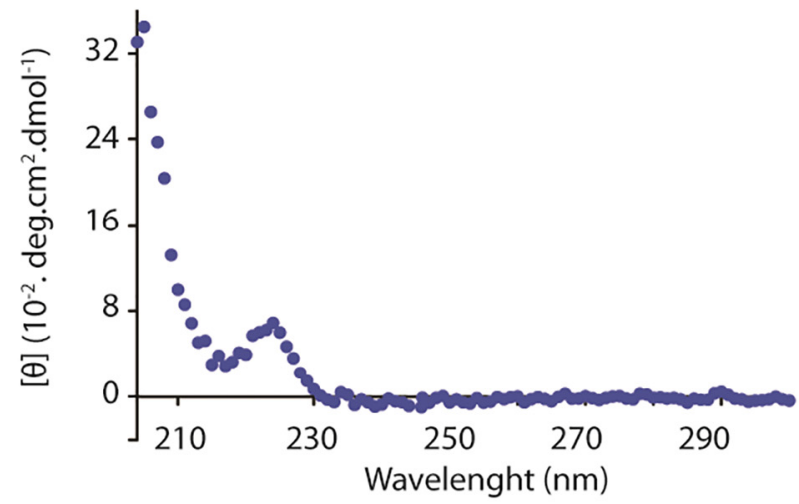

(c)

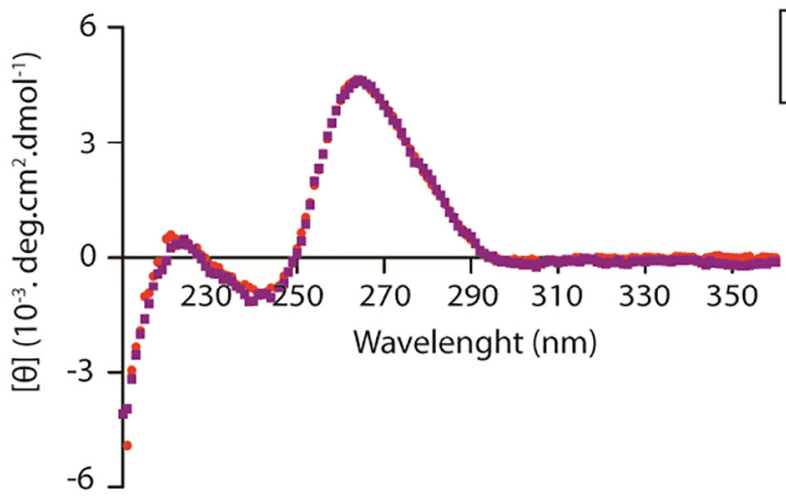

Fig. 10. $C D$ spectra recorded for the $\varphi B C 1-$ rib $_{g / m s}$ alone (a), $c A b_{B C 1 \text { rib } 3}$ alone (b) and the $c A b_{\text {BC1rib }} 3: \varphi B C 1$-rib ${ }_{\text {glms }}$ complex (c). The spectra measured for the nanobody and the stRNA alone were recorded with a 13- $\mu \mathrm{M}$ protein concentration and a 500-nM stRNA concentration, respectively. For the complex, the concentration of both the stRNA and the protein was $500 \mathrm{nM}$.

processes (Fig. 11c and Table 2) since the middenaturation upon heating and cooling $(\mathrm{Tm})$ for both processes is very similar to those measured for $\varphi B C$ 1-rib $_{g / m s}$ alone. Altogether, this result suggests that $\mathrm{CAb}_{\mathrm{BC} 1 \mathrm{rib}} 3$ does not modify the unfolding and refolding behavior of the stRNA upon thermal denaturation.

Reversibility of the thermal denaturation monitored using far UV $C D$

The CD spectrum recorded after a complete denaturation/renaturation cycle superimposes the one recorded at the start of the experiment before sample denaturation (Fig. 12), suggesting that the thermal denaturation is reversible.

\section{Discussion}

The overall goal of this study was to develop camelid antibody fragments or nanobodies able to bind with high affinity and specificity to a particular stRNA. Such nanobodies could be employed as innovative tools to investigate the structure and function of the nonprotein coding part of genomes. Our strategy involved the construction of a synthetic library of nanobodies, in which 11 residues ( 3 from CDR1 and 8 from CDR3) 
(a)

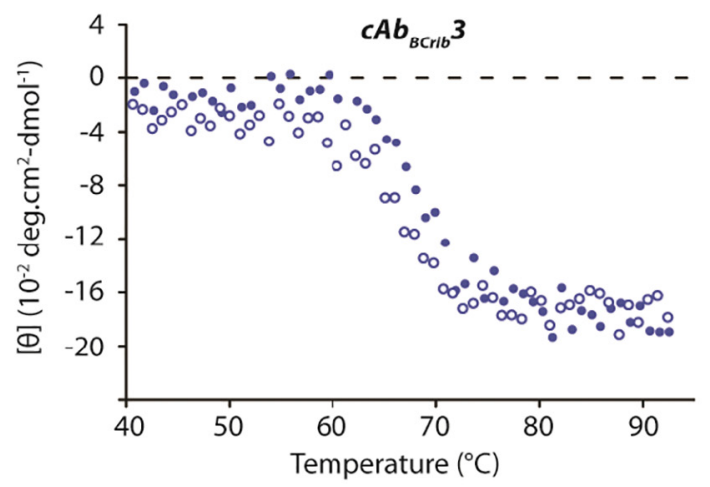

(c)

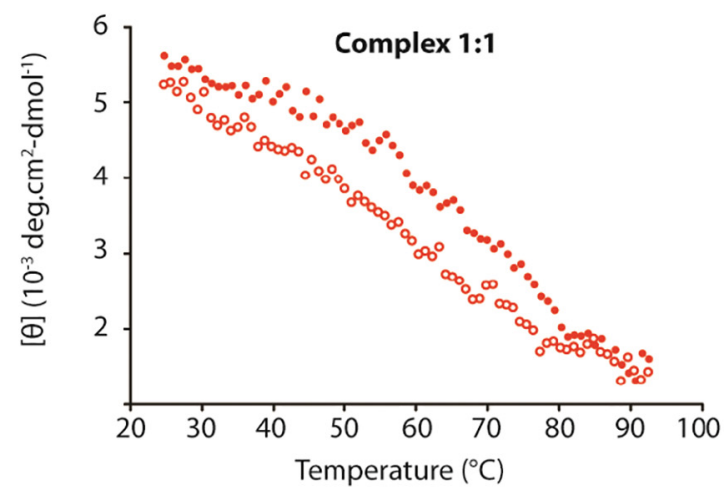

( (b)

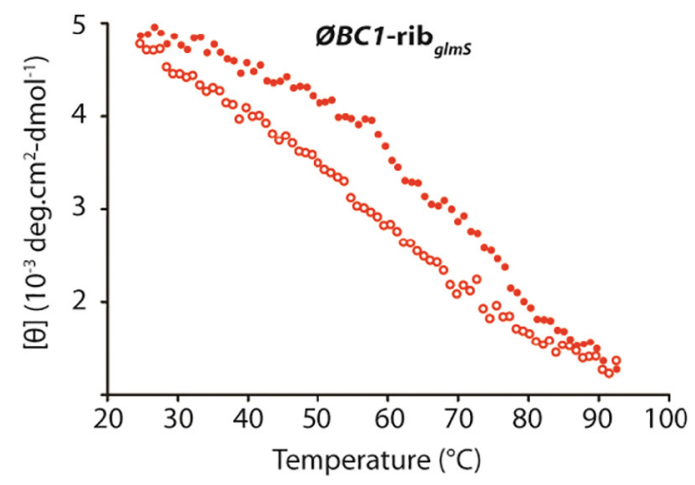

Fig. 11. Thermal denaturation curves (full circles) and renaturation curves (empty circles) measured for the $\mathrm{cAb}_{\mathrm{BC} 1 \mathrm{rib}} 3$ (a) and $\varphi B C 1-$ rib $_{\text {glms }}$ alone (b) or in complex with the nanobody using a 1:1 molar ratio (c). These curves were obtained by monitoring the CD signal at $223 \mathrm{~nm}$ for the nanobody and $267 \mathrm{~nm}$ for the stRNA and the complex, while heating the samples from 25 to $92{ }^{\circ} \mathrm{C}$ (denaturation) and then cooling the samples from 92 to $25^{\circ} \mathrm{C}$, at a heating and cooling rate of $1{ }^{\circ} \mathrm{C} / \mathrm{min}$.

were randomized. This randomization introduced a bias in favor of 6 residues (Arg, Lys, Tyr, Ser, Gly, Asn) that are often found to interact with RNA in protein:RNA complexes. The first panning of this library by phage

Table 2. Melting temperature values (Tm) calculated from the thermal denaturation and renaturation curves monitored by CD

\begin{tabular}{lcc}
\hline & $\begin{array}{c}\text { Tm denaturation } \\
\left({ }^{\circ} \mathrm{C}\right)\end{array}$ & $\begin{array}{c}\text { Tm renaturation } \\
\left({ }^{\circ} \mathrm{C}\right)\end{array}$ \\
\hline$\varphi B C 1-\mathrm{rib}_{\text {glms }}$ alone & $70.7 \pm 2.2$ & $62.0 \pm 4.0$ \\
$\varphi B C 1-\mathrm{rib}_{\text {glms }}: \mathrm{CAb}(1: 1)$ & $70.3 \pm 0.9$ & $62.0 \pm 4.2$ \\
\hline
\end{tabular}

The $\mathrm{Tm}$ values for denaturation and renaturation were measured for $\varphi B C 1-$ rib $_{\text {glms }}$ alone or in complex with the nanobody (1:1 molar ratio). The transition curves were all fitted using a sigmoidal dose response (variable slope) equation (see Materials and Methods). The presented $\mathrm{Tm}$ values and associated errors correspond to averages and standard deviations calculated from, at least, 3 independent experiments. display led to the selection of two individual variants ( $\mathrm{CAb}_{\mathrm{BC} 1 \text { rib3 }}$ and $\left.\mathrm{CAb}_{\mathrm{BC} 1 \text { rib90 }}\right)$ that showed strong binding to stRNA using the BLI technology. These data indicate that this library of camelid antibody fragments optimized for RNA interactions is a valid approach to select nanobodies able to associate with an RNA target and potentially other nucleic acidderived molecules.

Importantly, up to date, only a couple of studies have reported the successful selection of conventional antibody fragments (Fabs) that bind either stRNAs [51,52] or ssRNAs [53] in both cases also using a phage-display based selection approach. In these studies, the authors used two different synthetic Fab libraries harboring a "reduced genetic code." In one of these libraries, named YSGR, the entire sequence of light-chain CDR-3 (CDR-L3), and heavy-chain CDR-1, -2 and -3 (CDR-H1, CDR-H2, CDR-H3) were mutated in Tyr, Ser, Gly or Arg, whereas in the second library, termed YSGRKX, the entire sequences of the 6 CDRs were mutated in Tyr, Ser, Gly, Arg, Lys, and (X) all the 
(a)

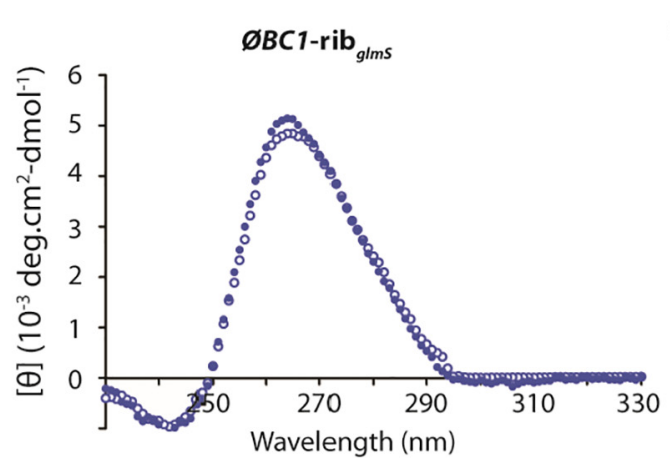

(b)

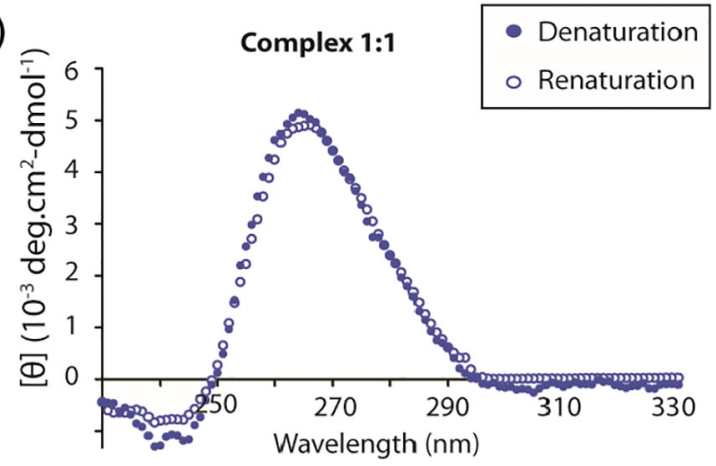

Fig. 12. $C D$ spectra recorded for $\varphi B C 1-$ rib $_{\text {glms }}$ alone (a) and the $C A b_{B C 1 \text { rib }} 3: \varphi B C 1-$ rib $_{\text {glms }}$ complex at a 1:1 molar ratio (b). These spectra were recorded before denaturation (full circles) and after renaturation (empty circles) with a 500-nM stRNA concentration. For the complex, the stRNA and protein concentrations used were $500 \mathrm{nM}$.

other residues except Cys, lle and Met. Although these studies were successful and led to the co-crystallization of a Fab:stRNA complex, it is interesting to note that most of the selected Fabs from these libraries exhibited a poor binding affinity in the low $\mu \mathrm{M}(\geq 1.5 \mu \mathrm{M})$ to high $\mathrm{nM}(\geq 160 \mathrm{nM})$ range compared to the nanobodybased binder (low nM) described in the present study. This is remarkable since the randomized surface in the Fab libraries was much larger than that in the nanobody library. This observation suggests that by mutating 11 residues only, across CDR1 and CDR3, it is possible to obtain tight binders against the target molecule instead of sampling a larger sequence space from libraries with more randomized residues.

This high binding affinity constitutes a strong advantage for co-crystallization experiments, given that nanobodies, like Fabs, are commonly used as chaperones in order to facilitate the crystallization of difficult protein targets (e.g., membrane proteins, etc...) $[25,54]$. In addition, this strong binding is also interesting for functional studies in cultured cells (in vitro) or in animal models (in vivo).

Furthermore, in contrast to $c A b_{\mathrm{BC} 1 \mathrm{rib}} 3$, some of the Fabs discussed above have a better differential binding specificity for particular stRNAs. A possible explanation for their enhanced discrimination among various stRNAs might originate from the use of competitor molecules, such as transfer RNAs (tRNAs) mixtures, during the phage display panning. The addition of increasing amounts of such competitors during the consecutive phage display selection rounds probably assisted in the selection of variants with higher specificity. The strategy of including competitor molecules during the selections could certainly be introduced in future pannings of our nanobody library to improve the binding specificity of the retrieved nanobodies.

Without any doubt, the binding specificity is a crucial parameter when designing antibody fragments for any sort of applications. Unlike proteins that are composed of 20 possible subunits (amino acids) and therefore presenting a large chemical diversity, RNAs include only four different subunits (nucleotides). Consequently, for molecules of similar size, the chemical diversity of RNA epitopes is much lower compared to protein epitopes. This RNA property probably explains why it is more challenging to obtain a high specificity for RNA antigens compared to protein antigens. Nevertheless, $\mathrm{CAb}_{\mathrm{BC} 1 \mathrm{rib}} 3$ exhibits clear target specificity since no association (or very weak) could be detected with ssRNA, ssDNA, dsRNA, dsDNA or BSA, demonstrating that it is specific for stRNA. In addition, $c A b_{B C 1 r i b} 3$ also discriminates among stRNAs since it does not recognize the isolated $\mathrm{rib}_{\mathrm{glms}}$.

Furthermore, our data also indicated that $\mathrm{CAb}_{\mathrm{BC} 1 \mathrm{rib}} 3$ can also discriminate $\varphi B C 1$ from its cousin $B C 1$, although these two stRNAs present a high degree of sequence identity in the $5^{\prime}$ domain (sequence identity of $92 \%$ ) (Fig. 1). In contrast, the central polyA and the $3^{\prime}$ domains deviate significantly between $B C 1$ and $\varphi B C 1$, with sequence identities of only $44 \%$ and $54 \%$, respectively. In order to explain the discrimination of $\mathrm{CAb}_{\mathrm{BC} 1 \mathrm{rib}} 3$ for $\varphi B C 1$ over $B C 1$, we have analyzed their secondary structure contents. Experimental determination of the secondary structure content of $B C 1$ [23] revealed that both the $5^{\prime}$ and $3^{\prime}$ regions form an hairpin, whereas the central polyA region is unpaired [22]. The secondary structure prediction performed on $\varphi B C 1$ using the RNAfold server (http://rna.tbi.univie.ac.at/ cgi-bin/RNAWebSuite/RNAfold.cgi) from the Vienna RNA Web services website (http://rna.tbi.univie.ac.at), indicates that the $5^{\prime}$ domain of $\varphi B C 1$ also forms a hairpin similar to that of $B C 1$, whereas the central poly $A$ and $3^{\prime}$ domains are predicted to be unpaired. Since our data showed that $c A b_{B C 1}$ rib3 fails to recognize ssRNA, such as the unpaired central poly $A$ and $3^{\prime}$ domain of $\varphi B C 1$, it is therefore more likely that $C A b_{B C 1 \text { rib }} 3$ recognizes the $5^{\prime}$ hairpin of $\varphi B C 1$. Notably, we hypothesized that the $5^{\prime}$ domain of $B C 1$ contains subtle conformational or dynamic changes 
that might reshape the epitope surface, leading to lower binding efficiency compared to $\varphi B C 1$, although at this stage, we consider the investigation of the bases causing this differential binding between $B C 1$ and $\varphi B C 1$ to be outside the scope of this study. Nevertheless, this differential binding between homologous stRNAs is important because it demonstrates the discriminating potential of nanobodies among various stRNAs.

In this work, we have also observed a strong increase in the binding affinity $(100 \times)$ between $\mathrm{CAb}_{\mathrm{BC} 1 \mathrm{rib}} 3$ and $\varphi B C 1$ (isolated or in the fusion) in the absence of $\mathrm{Mg}^{2+}$ compared to the affinity measured in its presence. By chelating $\mathrm{Mg}^{2+}$ ions, EDTA destabilizes the RNA tertiary structure, while the secondary structure content is maintained. Therefore, we postulate that $\mathrm{CAb}_{\mathrm{BC} 1 \text { rib }} 3$ recognizes one secondary structure element that is more accessible in the absence of $\mathrm{Mg}^{2+}$. This would explain the strong increase in affinity observed in the presence of EDTA.

At this stage, it remains difficult to describe the structural details of the interaction between $\mathrm{cAb}_{\mathrm{BC} 1 \text { rib }} 3$ and $\varphi B C 1$. We performed isothermal titration calorimetry experiments, microscale thermophoresis and gel filtration experiments in an attempt to explain the BLI data and determine other parameters such as the stoichiometry as well as the thermodynamic parameters $(\Delta H, \Delta S)$ of this interaction. Unfortunately, none of these methods led to conclusive and exploitable data. Nevertheless, the overall results generated in this study suggest that $\mathrm{CAb}_{\mathrm{BC} 1 \mathrm{rib}} 3$ recognizes a secondary structure element that is probably highly similar to the one included in the $5^{\prime}$ domain of $\varphi B C 1$ and common to the different stRNAs that were shown to interact with $c A b_{\mathrm{BC} 1 \mathrm{rib}} 3$.

The CD spectra of RNA alone and in the complex with the nanobody are virtually identical. This observation suggests that the binding of the nanobody does not affect the RNA secondary structure content of the RNA partner, which is an important property in the context of nanobody:stRNA co-crystallization experiments. In addition, our CD studies have also demonstrated that $c \mathrm{Ab}_{\mathrm{BC} 1 \mathrm{rib}} 3$ is a thermostable protein which is also advantageous for functional studies performed in cultured cells or animal models.

In conclusion, this study describes for the first time the successful design of a camelid antibody fragment able to recognize stRNA with high affinity retrieved from an innovative nanobody library dedicated for nucleic acid targets. Although the binding specificity of the obtained nanobody might be improved, this study represents a major step toward our long-term ambitious goal that aims to provide innovative tools for functional and structural RNA studies. Conversely, this lack of strict specificity can be advantageous since $\mathrm{CAb}_{\mathrm{BC} 1 \mathrm{rib}} 3$ could potentially be used to assist the crystallization of several stRNA targets. This aspect will be the next focus of future investigations.

\section{Materials and Methods}

\section{Nanobody library construction}

The synthetic nanobody gene library harboring the 11 randomized residues in CDR1 and CDR3 regions was purchased from GeneArt with an estimated diversity of $\approx 10^{11}$ individual gene variants. The library was amplified using the following specific primers that included the Pst1 and Not1 restriction enzyme sites (underlined):

\section{Fwd: 5'-GTTCAGCTGCAGGAAAGCGGTGGTG GTAGCGTTC-3'}

Rev.: 5'-CGGGTAGCGGCCGCTCGAAACG-3'

These primers anneal to the framework 1 and framework 4 regions. The final and purified PCR product was digested with Not1 and Pst1, purified and then cloned into the phagemid vector pHEN4 using the protocol described by $\mathrm{Hu}$ et al. [55]. Briefly, the ligation of the nanobody gene library into the pHEN4 was performed at $16{ }^{\circ} \mathrm{C}$ for $16 \mathrm{~h}$, and the ligation product was purified and then used to transform, by electroporation, TG1 electro-competent cells (Lucigen, Middleton, WI, USA). Immediately after the electrotransformation, the cells were allowed to recover with SOC medium, by incubation at $37^{\circ} \mathrm{C}$ for $1 \mathrm{~h}$. The transformed cells were then plated on LB/agar plates containing $2 \%$ glucose and $100 \mu \mathrm{g} / \mathrm{ml}$ ampicillin. Serial dilutions of cell aliquot were plated on standard-sized Petri dishes to determine the total amount of transformants, while the majority of the cells was plated on large Petri dishes. The plates were incubated overnight at $37^{\circ} \mathrm{C}$, and the colonies were scraped from the plates and stored at $-80^{\circ} \mathrm{C}$ in LB/ $20 \%$ glycerol at $-80^{\circ} \mathrm{C}$ for future use. The percentage of clones harboring a phagemid containing an insert corresponding to the size of a nanobody gene was assessed by colony PCR using the MP57 and GIll primers on 95 randomly chosen colonies.

\section{MP57: 5'-TTA TGC TTC CGG CTC GTA TG-3' \\ GIII: 5'-CCA CAG ACA GCC CTC ATA G-3'}

These primers anneal on both sides on the pHEN4 vector sequences abutting the inserted nanobody gene.

\section{Display of the nanobody library on phage particles and selection of binders}

The nanobodies within the library were expressed on phage particles after infection with M13 K07 helper phages. Enrichment of binders from this library was performed by five rounds of in vitro selection using a protocol adapted from Hu and coworkers [55]. Briefly, 
an E. coliTG1 cell aliquot of the nanobody library was cultured in $300 \mathrm{ml}$ of 2 XTY medium supplemented with $1 \%(\mathrm{w} / \mathrm{v})$ glucose and $100 \mu \mathrm{g} / \mathrm{ml}$ ampicillin until the $\mathrm{OD}_{600}$ value reached $0.6-0.9$. Then, the cells were infected with $\sim 10^{12} \mathrm{M} 13$ helper phage particles for $30 \mathrm{~min}$ at room temperature (RT). The infected cells were harvested by centrifugation at $1300 \mathrm{~g}$ for $10 \mathrm{~min}$ and resuspended in $300 \mathrm{ml}$ fresh 2xTY medium supplemented with $100 \mu \mathrm{g} / \mathrm{ml}$ ampicillin and $70 \mu \mathrm{g} / \mathrm{ml}$ kanamycin to secrete virions displaying nanobodies fused the coat protein plll. After overnight growth, bacterial cells were removed by centrifugation and the phages particles secreted in the culture supernatants were precipitated using a polyethylene glycol/ $\mathrm{NaCl}$ solution. The phage solution was re-suspended in a total volume of $1 \mathrm{ml}$ sterile PBS and the phage concentration was calculated based on the $\mathrm{OD}_{260 \mathrm{~nm}}$ value. For the selection rounds, $60 \mu \mathrm{g}$ of purified and in vitro transcribed $\varphi B C 1$-rib $_{\text {glms }}$ was coated overnight at $4{ }^{\circ} \mathrm{C}$ in a single well of a microtiter plate. Blocking of the stRNA-coated well and the non-coated control well (located at a distant position in the microtiter plate) was performed with different blocking solutions for the successive selection rounds. These blocking solution include the following: $4 \%(\mathrm{w} / \mathrm{v})$ skimmed-milk proteins, protein free buffer (Thermo Scientific), $0.1 \%$ casein (w/v) or SEA BLOCK blocking buffer (Thermo Scientific). Changing of the blocking solution minimizes enrichment of phages that bind to components present in the blocking reagent. For each selection round, $\sim 10^{11}$ phage particles were added per well and incubated for $1 \mathrm{~h}$ at RT. Increasing numbers of washings were performed with PBST (PBS containing $0.05 \%$ Tween-20) during the successive selection rounds. The elution of phages particles bound to the stRNA-coated well was performed with a triethylamine solution $(100 \mathrm{mM}$ TEA, pH 11.0) for $10 \mathrm{~min}$ at RT. The eluted phages were immediately neutralized with Tris $-\mathrm{HCl}(1.0 \mathrm{M}$, $\mathrm{pH}$ 7.4). Aliquots of the eluted phage solutions from each well (coated or not coated with stRNA) were serially diluted $(10 x)$ and used to infect exponentially growing TG1 cells. After 30-min incubation at RT, infected cells for each phage dilution were plated on selective medium to assess relative phage enrichment between the stRNA-coated and non-coated wells. The majority of the stRNA-eluted phages was also used to infect exponential growing $E$. coli TG1 cells in order to amplify and prepare phage particles for the next selection round. The panning was performed for five consecutive rounds of selection.

\section{Screening of stRNA binders by ELISAs}

Ninety-five individual colonies from rounds 3,4 and 5 were randomly picked from the plates and grown in $100 \mu \mathrm{l}$ of 2 XTY medium supplemented with $10 \%$ glycerol, $2 \%$ glucose and $100 \mu \mathrm{g} / \mathrm{ml}$ ampicillin in a 96-microwell plate (round bottom). After overnight culture at $37^{\circ} \mathrm{C}, 10 \mu \mathrm{l}$ of each of these cultured colony was used to inoculate $2 \mathrm{ml}$ of 2xTY medium supplemented with $0.1 \%$ glucose and $100 \mu \mathrm{g} / \mathrm{ml}$ ampicillin in a 96-microwell plate (deep well). The leftover from the overnight pre-culture was frozen and stored at $-20^{\circ} \mathrm{C}$ for subsequent experiments. The 2-ml deep-well culture plates were incubated for $4-5 \mathrm{~h}$ at $37^{\circ} \mathrm{C}$ with shaking before inducing the expression of the nanobodies in fusion with the phage coat protein pIII (Nterminal end) and an HA tag (C-terminal end) by the addition of $1 \mathrm{mM}$ IPTG. After $4 \mathrm{~h}$ of incubation at $37^{\circ} \mathrm{C}$, the cultures were harvested by centrifugation, and the cell pellets were frozen at $-20^{\circ} \mathrm{C}$. The nanobodies exported in the periplasmic space were extracted by freeze-thaw cycles and used in an ELISA to detected nanobody variants able to bind $\varphi B C 1-$ $\mathrm{rib}_{\mathrm{glms}}$ coated in a 96-microwell plate. Each soluble extract was added to a well coated with $100 \mu \mathrm{l} \varphi B C 1-$ $\mathrm{rib}_{\text {glms }}$ at $70 \mu \mathrm{g} / \mathrm{ml}$ as well as a negative control well (non-coated well). Nanobodies able to bind stRNA were detected with 1/2000 diluted mouse anti-HA IgG (primary antibody) and a 1/2000 goat anti-mouse IgG conjugated with alkaline phosphatase (secondary antibody) (ImTec Diagnostics NV, Antwerp, Belgium). After the addition of the substrate $p$-nitrophenyl phosphate (Sigma), the reaction was measured after $5,15,30$ and $60 \mathrm{~min}$ at $410 \mathrm{~nm}$. The colonies expressing nanobodies that exhibit a positive signal in the ELISA were cultured. The corresponding phagemid DNAs were extracted and purified, and the inserted nanobody genes were sequenced.

\section{Expression of the nanobodies from the pHEN4 phagemid vector and periplasmic extract preparation}

The gene of nanobodies corresponding to clones $\mathrm{CAb}_{\mathrm{BC} 1 \mathrm{rib}} 3, \mathrm{CAb}_{\mathrm{BC} \text { rib }} 20$ and $\mathrm{cA} \mathrm{b}_{\mathrm{BC} \text { 1rib }} 90$ cloned into the phagemid vector pHEN4 were transformed into $E$. coli WK6 cells for expression of the nanobodies fused to an HA tag on their C-terminal end. Recombinant expression of the nanobodies was performed in shake flasks using Terrific broth supplemented with $100 \mu \mathrm{g} / \mathrm{ml}$ ampicillin until the $\mathrm{OD}_{600 \mathrm{~nm}}$ reaches $\approx 0.8$. Then, induction of protein expression was performed by adding IPTG (1 mM) and incubating for $16 \mathrm{~h}$ at $28^{\circ} \mathrm{C}$. After pelleting the cells, the periplasmic proteins were extracted by osmotic shock [56]. The periplasmic extracts were then dialyzed in Tris $50 \mathrm{mM}(\mathrm{pH} 7.5)$, $\mathrm{NaCl} 150 \mathrm{mM}$ and $10 \mathrm{mM} \mathrm{MgCl}_{2}$ for the binding assays performed using $\mathrm{BLI}$.

\section{Expression and purification of the nanobodies expressed from the pHEN6 plasmid}

The nanobody genes corresponding to clones $c A b_{\mathrm{BC} 1 \mathrm{rib}} 3$ and $c A b_{\mathrm{BC} 1 \text { rib }} 90$ were sub-cloned into the expression vectors pHEN6 using the restriction 
enzyme sites Ncol and BstEll. The plasmid constructs were transformed into E. coli WK6 cells for nanobody expression. Production of the recombinant nanobodies was performed in shake flasks using Terrific broth supplemented with $100 \mu \mathrm{g} / \mathrm{ml}$ ampicillin until the $\mathrm{OD}_{600 \mathrm{~nm}}$ reaches $\approx 4$. Then, induction of protein expression was performed by the addition of $1 \mathrm{mM}$ IPTG for $16 \mathrm{~h}$ at $28^{\circ} \mathrm{C}$. The cells were harvested by centrifugation and the pellets were resuspended in $50 \mathrm{mM}$ Tris (pH 7.5), $150 \mathrm{mM} \mathrm{NaCl}$ and $10 \mathrm{mM}$ imidazole. Total cell lysis was performed on an EmulsiFlex-C3 (Avestin). The soluble fraction of cell lysate was recovered and loaded on a Ni-nitrilotriacetic acid column (Qiagen), and after washing, the bound proteins were eluted with a $500 \mathrm{mM}$ imidazole buffer. The purity of the protein sample was further increased using a cationic exchange chromatography. Briefly, the nanobody eluted from the first purification step was dialyzed overnight at $4{ }^{\circ} \mathrm{C}$ in a low salt buffer [50 mM Hepes (pH 7), $20 \mathrm{mM} \mathrm{NaCl}$ ) and then loaded onto a 1 ml Mono-S (5/50 GL, GE Healthcare) column. The protein was eluted using a linear gradient performed with a 1-M NaCl buffer. The eluted fractions were dialyzed against $50 \mathrm{mM}$ Hepes $(\mathrm{pH} 7)$ and $150 \mathrm{mM}$ $\mathrm{NaCl}$ and then concentrated to $\approx 15 \mu \mathrm{M}$ before storage at $-20^{\circ} \mathrm{C}$. The final protein concentration was determined from the UV absorption at $280 \mathrm{~nm}$ using the theoretical extinction coefficient of the nanobody calculated based on its amino acid sequence. The purity and integrity of the purified nanobody sample were checked using Coomassie-stained SDS-PAGE and mass spectrometry (ESI-Q-ToF) under reducing and non-reducing conditions in order to check the presence of the stabilizing disulfide bond between Cys 22 and Cys 96 (IMGT numbering scheme).

\section{stRNA genetic constructs}

The genes encoding the different stRNAs cloned downstream the T7 promoter sequence into the pUC57 plasmid in between the restriction sites BamH1 ( $5^{\prime}$ end of the genes) and EcoR1 ( $3^{\prime}$ end of the genes) were purchased from GeneCust (Luxembourg). Notably, for the RNA fusion $\varphi B C 1-$ $\mathrm{rib}_{\mathrm{glms}}$, we added a stretch of 10 adenines between $\varphi B C 1$ and rib $_{\text {glms }}$ to prevent any steric hindrances. The stRNA gene sequences used in this study are detailed below:

$\varphi B C 1:$

GAAATTAATACGACTCACTATAGGGAAG GAGTTGGGGATTTAGCTCAGTGGTAG A A T G C T T G C C T A G C A A G C G C A AGGCCCTGGGTTCAGTCCTCAGCTCTGT GAAAGGAAAAAAAAAGACAAAATAAATAATT TAGGAAGTATATGTTAATTGAGGGAAAGAA TATAAGTAAATCAAATTACATCATTATT.
$B C 1:$

GAAATTAATACGACTCACTATAGGG GGGGTTGGGGATTTAGCTCAGTGGTA GAGCGCTTGCCTAGCAAGCGCAAGG CCCTGGGTTCGGTCCTCAGCTCTGGAAA AAAAAAAAAAAAAAAAAAAAGACAAAAT AACAAAAAGACCAAAAAAAAACAAGG TAACTGGCACACACAACCTTT.

Rib ${ }_{g / m s}:$

GAAATTAATACGACTCACTATAGGG A A GCGCCAGA A C T G G C A C A T T G CACTCCGGTGCCAGTTGACGAGGTGGGG TTTATCGAGATTTCGGCGGATGACTCCC GGTTGTTCATCACAACCGCAAGCTTTTACT TAAATCATTAAGGTGACTTAGTGGA C A A A G G T G A A A T G T G A T G A C G AGCAGGCCTGCGGAAT.

Fusion RNA $\varphi B C$ 1-rib $_{\text {glms: }}$ :

GAAATTAATACGACTCACTATAGGGAAG GAGTTGGGGATTTAGCTCAGTGGTAGAAT GCTTGCCTAGCAAGCGCAAGGCCCTGGGTT C A G T C C T C A G C T C T G T G A A AGGAAAAAAAAGACAAAATAAATAA TTTAGGAAGTATATGTTAATTGAGGGAAA GAATATAAGTAAATCAAATTACATCATTAT TAAAAAAAAAAAAAAAGGATCCAAGCGCCA GAACTGGCACCATTGCACTCCGGTGC CAGTTGACGAGGTGGGGTTTATCGA GATTTCGGCGGATGACTCCCGGTTGTTCAT CACAACCGCAAGCTTTTACTTAAATCAT TAAGGTGACTTAGTGGACAAAGGTGAAAGTG TGATGACGAGCAGGCCTGCGGAAT.

stRNA ctrl 1: 7SK: the human small nuclear RNA. GAAATTAATACGACTCACTATAGGGGGATGT GAGGGCGATCTGGCTGCGACA TCTGT CACCCCATTGATCGCCAGGGTTGATTCGGCT GATCTGGCTGGCTAGGCGGGTGTCCCCTT CCTCCCTCACCGCTCCATGTGCGTCCCTCCC GAAGCTGCGCGCTCGGTCGAAGAGGACGAC C A T C C C C G A TA G A G G A G G A C C G G T CTTCGGTCAAGGGTATACGAGTAG CTGCGCTCCCCTGCTAGAACCTCCAAA CAAGCTCTCAAGGTCCATTTGTAGGAGAACGTAGGGTAGTCAAGCTTCCAAGACTCCAGACACATCCAAATGAGGCGCTGCATGTGG CAGTCTGCCTTTCTTTT. 
stRNA ctrl 2: P4P6 domain of Tetrahymena group I catalytic intron.

G A A A T TA A TACGACTCACTATAGGG GGAATTGCGGGAAAGGGGTCAACAGCCGTT CAGTACCAAGTCTCAGGGGAAACTTTGA GATGGCCTTGCAAAGGGTATGGTAA TAAGCTGACGGACATGGTCCTAACACG CAGCCAAGTCCTAAGTCAACAGATCTTCT GTTGATATGGATGCAGTTCA.

\section{stRNA sample preparation}

All the in vitro transcribed stRNAs used in this study were produced by linearizing the correspond-

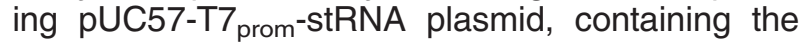
gene encoding the stRNA of interest downstream the T7 promoter sequence and harboring an EcoR1 restriction site at its $3^{\prime}$ end. Linearization of the plasmid with EcoR1 results in a $5^{\prime}$ overhang that was used to terminate the transcription reaction after the stRNA gene. Transcription was carried out using the in vitro transcription kit (RiboMAX ${ }^{\mathrm{TM}}$ Large Scale RNA production system-T7, Promega) following the manufacturer's instructions. The transcription product was purified and the unincorporated nucleotides removed using mini Quick Spin RNA columns (Roche). The final stRNA concentration was determined from the UV absorption at $260 \mathrm{~nm}$, using the theoretical extinction coefficient of the stRNA calculated based on its nucleotide sequence. The transcripts were stored at $-80{ }^{\circ} \mathrm{C}$ until needed.

To check the quality of the transcription product, ribozyme catalytic activity measurements were performed. Briefly, $\varphi B C 1$-rib $_{g I m s}(3 \mu \mathrm{M})$ was incubated at $37^{\circ} \mathrm{C}$ for $30 \mathrm{~min}$ in the presence of $600 \mu \mathrm{M}$ GlcN6P, in $50 \mathrm{mM}$ Hepes (pH 7.5), $150 \mathrm{mM} \mathrm{NaCl}$ and $10 \mathrm{mM} \mathrm{MgCl} 2$, in a final volume of $16 \mu \mathrm{l}$. The reaction was stopped by adding dimethyl sulfoxide $80 \%(\mathrm{v} / \mathrm{v})$. The sample was analyzed by electrophoresis on a $2 \%$ agarose gel supplemented with an intercalating agent (Midori Green DNA stain) to detect the RNA bands (data not shown). This experiment allows us to assess the homogeneity and integrity of the sample. In addition, although there is no way to assess the proper folding of the $\varphi B C 1$ moiety of the RNA fusion, the presence of a cleavage product indicates that the transcription was performed in conditions compatible with the native conformation of an stRNA.

\section{Binding assays using BLI}

$\mathrm{BLI}$ experiments were carried out at $30^{\circ} \mathrm{C}$ in 96-well black polypropylene microplates (Greiner), with agitation set to $1000 \mathrm{rpm}$, on an Octet HTX instrument (FortéBio, Pall). All the protein and RNA solutions were diluted in the experimental buffer and supplemented with RNase inhibitor $(0.4 \mathrm{U} / \mu \mathrm{l})$
(Ribosafe RNase Inhibitor, Bioline) to ensure RNase-free conditions.

The binding assays performed on the periplasmic extracts containing the nanobodies were carried out in $50 \mathrm{mM}$ phosphate saline buffer (pH 7.5), $140 \mathrm{mM}$ $\mathrm{NaCl}$ and $10 \mathrm{mM} \mathrm{MgCl} 2$ supplemented with $0.1 \%$ BSA and $0.02 \%$ Tween-20. The binding experiments were performed by immobilizing a $1 \mathrm{\mu g} / \mathrm{ml}$ anti-HA tag mouse antibody (Abcam ab18181) solution on antimouse IgG Fc capture biosensors (AMC, Pall) for $200 \mathrm{~s}$. A baseline was then recorded for $60 \mathrm{~s}$ in the experimental buffer before loading the periplasmic extracts for $600 \mathrm{~s}$ to allow for immobilization of the nanobodies harboring a C-terminal HA-tag. A second baseline was then monitored for 120 s before recording the analyte (stRNA) association for $600 \mathrm{~s}$ with a 1- $\mu \mathrm{M}$ stRNA concentration. The dissociation was measured for $600 \mathrm{~s}$ in the experimental buffer.

The binding assays performed on the purified nanobodies were carried out in $50 \mathrm{mM}$ Hepes (pH 7.5), $150 \mathrm{mM} \mathrm{NaCl}, 10 \mathrm{mM} \mathrm{MgCl}_{2}$ or EDTA, supplemented with $0.1 \%$ BSA and $0.02 \%$ Tween-20 in order to minimize nonspecific interactions. All the binding experiments were carried out by immobilizing the purified $\mathrm{CAb}_{\mathrm{BC} 1 \mathrm{rib}} 3$ harboring a $\mathrm{C}$-terminal $\mathrm{His}_{\mathrm{x} 6}$ tag at a concentration of $1 \mu \mathrm{g} / \mathrm{ml}$ on anti-His-coated biosensors (HIS1K, Pall) for $120 \mathrm{~s}$. A baseline was then monitored for $300 \mathrm{~s}$ in the experimental buffer. For the qualitative binding assays, binding against the tested stRNAs was recorded for $600 \mathrm{~s}$ using an RNA concentration of $100 \mathrm{nM}$. The dissociation was recorded in the experimental buffer for $600 \mathrm{~s}$. For the quantitative binding assays, we recorded the binding for different stRNA concentrations ranging from 100 to $8.3 \mathrm{nM}$ (1.5-fold dilution series) in the presence of $10 \mathrm{mM} \mathrm{MgCl} 2$ or $10 \mathrm{mM}$ EDTA. In all cases, binding was measured for $60 \mathrm{~s}$ and dissociation in the experimental buffer for $300 \mathrm{~s}$. Recorded data were corrected by subtracting the signals from a reference sensor immobilized with $\mathrm{cAb}_{\mathrm{BC} 1 \mathrm{rib}} 3$ (baseline drift). Data were analyzed using Octet software, version 8.0 (Pall) and fitted using a 1:1 interaction model and a global fit. Therefore, a single set of kinetic parameters was obtained for all tested concentrations by nonlinear least-squares fitting.

\section{CD measurements}

CD spectra were recorded with a Jasco J-810 spectropolarimeter equipped with a thermostatically cell holder from 210 to $360 \mathrm{~nm}$ in $50 \mathrm{mM}$ Tris or Hepes buffer (pH 7.5), $150 \mathrm{mM} \mathrm{NaCl}$ and $10 \mathrm{mM} \mathrm{MgCl}_{2}$ using RNA and protein concentrations of $\sim 500 \mathrm{nM}$ and $13 \mu \mathrm{M}$, respectively. Spectra were acquired at $25^{\circ} \mathrm{C}$ in a quartz cell with $0.1-\mathrm{cm}$ path length at a scan rate of $50 \mathrm{~nm} / \mathrm{min}$, with a 1-nm bandwidth and a 2-s response. The presented spectra correspond to the average of three accumulations, from which the buffer spectrum acquired under identical conditions was subtracted. 
The thermal denaturation transitions were monitored at 267 and $223 \mathrm{~nm}$ for the RNA and protein samples, respectively. Experimental conditions were kept as above, and the temperature was increased from 25 to $92{ }^{\circ} \mathrm{C}$ at a rate of $1^{\circ} \mathrm{C} / \mathrm{min}$. Data were acquired every $0.5^{\circ} \mathrm{C}$, with a 4-s integration time and a 1-nm bandwidth. Notably, we tested different rates of temperature increase $\left(0.2,0.5\right.$ and $\left.1{ }^{\circ} \mathrm{C} / \mathrm{min}\right)$. All the tested rates gave the same denaturation curves and $\mathrm{Tm}^{*}$, indicating that the measurements performed at $1{ }^{\circ} \mathrm{C} / \mathrm{min}$ were made at equilibrium. The transition curves were all fitted using a sigmoidal dose response (variable slope) equation: $Y=$ Bottom + (Top-Bottom)/ $\left(1+10^{\wedge}\left((\operatorname{LogEC} 50-X){ }^{*}\right.\right.$ HillSlope $\left.)\right)$, where $X$ is the temperature, $Y$ is the $C D$ signal intensity. $Y$ starts at the bottom and goes to the top using a sigmoid shape. LogEC50 is the Tm, namely the $X$ value when the response is halfway between the bottom and the top. Hillslope describes the steepness of the curve.

\section{Acknowledgments}

We thank the National Funds for Scientific Research of Belgium for funding most of the research and Dr. Ruth Kellner for providing assistance to design the graphical abstract. We also thank the Walloon Region (SPW, DGO6, Belgium) for supporting the ROBOTEINE project within the frame of the EQUIP2013 program, Convention 1318159. The salary of Julie Vandenameele is currently supported by the AUTOBMP2 project (SPW, DGO6, convention 16100518).

\section{Appendix A. Supplementary Data}

Supplementary data to this article can be found online at https://doi.org/10.1016/j.jmb.2018.03.032.

Received 12 January 2018; Received in revised form 8 March 2018;

Accepted 27 March 2018

Available online 11 April 2018

Keywords: nanobody; non-coding RNAs; structured RNAs;

RNA/protein interactions; antibody fragment engineering

$\dagger F$.C. and E.D. contributed equally to this work.

Abbreviations used: stRNA, structured RNA; ncRNA, non-coding RNA; cAb, camelid antibody fragment; RBP, RNA-binding protein.

\section{References}

[1] I. Dunham, et al., An integrated encyclopedia of DNA elements in the human genome, Nature 489 (2012) 57-74.

[2] J.S. Mattick, I.V. Makunin, Non-coding RNA, Hum. Mol. Genet. 15 Spec No. 1 (2006) R17-29.

[3] T.R. Cech, J.A. Steitz, The noncoding RNA revolution-trashing old rules to forge new ones, Cell 157 (2014) 77-94.

[4] P.J. Batista, H.Y. Chang, Long noncoding RNAs: cellular address codes in development and disease, Cell 152 (2013) 1298-1307.

[5] V.J. Peschansky, C. Wahlestedt, Non-coding RNAs as direct and indirect modulators of epigenetic regulation, Epigenetics 9 (2014) 3-12.

[6] M. Esteller, Non-coding RNAs in human disease, Nat. Rev. Genet. 12 (2011) 861-874.

[7] R.A. de Almeida, M.G. Fraczek, S. Parker, D. Delneri, R.T. O'Keefe, Non-coding RNAs and disease: the classical ncRNAs make a comeback, Biochem. Soc. Trans. 44 (2016) 1073-1078.

[8] I.V. Novikova, S.P. Hennelly, K.Y. Sanbonmatsu, Tackling structures of long noncoding RNAs, Int. J. Mol. Sci. 14 (2013) 23672-23684.

[9] D. Bhartiya, V. Scaria, Genomic variations in non-coding RNAs: Structure, function and regulation, Genomics 107 (2016) 59-68.

[10] E. Westhof, P. Auffinger, RNA tertiary structure, in: R.A. Meyers (Ed.), Encyclopedia of Analytical Chemistry, John Wiley \& Sons Ltd., Chichester 2000, pp. 5222-5232.

[11] V.K. Misra, D.E. Draper, On the role of magnesium ions in RNA stability, Biopolymers 48 (1998) 113-135.

[12] D.E. Draper, A guide to ions and RNA structure, RNA 10 (2004) 335-343.

[13] D. Grilley, A.M. Soto, D.E. Draper, $\mathrm{Mg}^{2+}$-RNA interaction free energies and their relationship to the folding of RNA tertiary structures, Proc. Natl. Acad. Sci. U. S. A. 103 (2006) 14003-14008.

[14] A. Filipovska, M.F. Razif, K.K. Nygard, O. Rackham, A universal code for RNA recognition by PUF proteins, Nat. Chem. Biol. 7 (7) (2011) 425.

[15] R. Choudhury, Y.S. Tsai, D. Dominguez, Y. Wang, Z. Wang, Engineering RNA endonucleases with customized sequence specificities, Nat. Commun. 3 (2012) 1147.

[16] A. Cooke, A. Prigge, L. Opperman, M. Wickens, Targeted translational regulation using the PUF protein family scaffold, Proc. Natl. Acad. Sci. U. S. A. 108 (2011) 15870-15875.

[17] J.A. Doudna, J.H. Cate, RNA structure: crystal clear? Curr. Opin. Struct. Biol. 7 (1997) 310-316.

[18] A.R. Ferre-D'Amare, K. Zhou, J.A. Doudna, A general module for RNA crystallization, J. Mol. Biol. 279 (1998) 621-631.

[19] A.Y. Keel, R.P. Rambo, R.T. Batey, J.S. Kieft, A general strategy to solve the phase problem in RNA crystallography, Structure 15 (2007) 761-772.

[20] R.C. Pink, K. Wicks, D.P. Caley, E.K. Punch, L. Jacobs, D.R. Carter, Pseudogenes: pseudo-functional or key regulators in health and disease? RNA 17 (2011) 792-798.

[21] E. Mus, P.R. Hof, H. Tiedge, Dendritic BC200 RNA in aging and in Alzheimer's disease, Proc. Natl. Acad. Sci. U. S. A. 104 (2007) 10679-10684.

[22] D. Lin, T.V. Pestova, C.U. Hellen, H. Tiedge, Translational control by a small RNA: dendritic BC1 RNA targets the eukaryotic initiation factor $4 \mathrm{~A}$ helicase mechanism, Mol. Cell. Biol. 28 (2008) 3008-3019. 
[23] T.S. Rozhdestvensky, A.M. Kopylov, J. Brosius, A. Huttenhofer, Neuronal BC1 RNA structure: evolutionary conversion of a tRNA (Ala) domain into an extended stem-loop structure, RNA 7 (2001) 722-730.

[24] D. Matelska, K. Steczkiewicz, K. Ginalski, Comprehensive classification of the PIN domain-like superfamily, Nucleic Acids Res. 45 (12) (2017) 6995-7020.

[25] S. Koide, Engineering of recombinant crystallization chaperones, Curr. Opin. Struct. Biol. 19 (2009) 449-457.

[26] T.A. Cooper, L. Wan, G. Dreyfuss, RNA and disease, Cell 136 (2009) 777-793.

[27] S. Muyldermans, Nanobodies: natural single-domain antibodies, Annu. Rev. Biochem. 82 (2013) 775-797.

[28] M.M. Harmsen, H.J. De Haard, Properties, production, and applications of camelid single-domain antibody fragments, Appl. Microbiol. Biotechnol. 77 (2007) 13-22.

[29] J. Wesolowski, V. Alzogaray, J. Reyelt, M. Unger, K. Juarez, M. Urrutia, A. Cauerhff, W. Danquah, B. Rissiek, F. Scheuplein, N. Schwarz, S. Adriouch, O. Boyer, M. Seman, A. Licea, D.V. Serreze, F.A. Goldbaum, F. Haag, F. Koch-Nolte, Single domain antibodies: promising experimental and therapeutic tools in infection and immunity, Med. Microbiol. Immunol. 198 (2009) 157-174.

[30] M. Unger, A.M. Eichhoff, L. Schumacher, M. Strysio, S. Menzel, C. Schwan, V. Alzogaray, V. Zylberman, M. Seman, J. Brandner, H. Rohde, K. Zhu, F. Haag, H.W. Mittrucker, F. Goldbaum, K. Aktories, F. Koch-Nolte, Selection of nanobodies that block the enzymatic and cytotoxic activities of the binary Clostridium difficile toxin CDT, Sci. Rep. 5 (2015) 7850.

[31] J.S. Sohier, C. Laurent, A. Chevigne, E. Pardon, V. Srinivasan, U. Wernery, P. Lassaux, J. Steyaert, M. Galleni, Allosteric inhibition of VIM metallo-beta-lactamases by a camelid nanobody, Biochem. J. 450 (2013) 477-486.

[32] R. Chakravarty, S. Goel, W. Cai, Nanobody: the "magic bullet" for molecular imaging? Theranostics 4 (2014) 386-398.

[33] O.Y. Dmitriev, S. Lutsenko, S. Muyldermans, Nanobodies as probes for protein dynamics in vitro and in cells, J. Biol. Chem. 291 (2016) 3767-3775.

[34] S. Harmansa, I. Alborelli, D. Bieli, E. Caussinus, M. Affolter, A nanobody-based toolset to investigate the role of protein localization and dispersal in Drosophila, eLife 6 (2017).

[35] K.E. Conrath, M. Lauwereys, M. Galleni, A. Matagne, J.M. Frere, J. Kinne, L. Wyns, S. Muyldermans, Beta-lactamase inhibitors derived from single-domain antibody fragments elicited in the camelidae, Antimicrob. Agents Chemother. 45 (2001) 2807-2812.

[36] C. Vincke, R. Loris, D. Saerens, S. Martinez-Rodriguez, S. Muyldermans, K. Conrath, General strategy to humanize a camelid single-domain antibody and identification of a universal humanized nanobody scaffold, J. Biol. Chem. 284 (2009) 3273-3284.

[37] D. Saerens, M. Pellis, R. Loris, E. Pardon, M. Dumoulin, A. Matagne, L. Wyns, S. Muyldermans, K. Conrath, Identification of a universal $\mathrm{VHH}$ framework to graft non-canonical antigen-binding loops of camel single-domain antibodies, J. Mol. Biol. 352 (2005) 597-607.

[38] M. Treger, E. Westhof, Statistical analysis of atomic contacts at RNA-protein interfaces, J. Mol. Recognit. 14 (2001) 199-214.

[39] E. Jeong, H. Kim, S.W. Lee, K. Han, Discovering the interaction propensities of amino acids and nucleotides from protein-RNA complexes, Mol. Cells 16 (2003) 161-167.

[40] D. Lejeune, N. Delsaux, B. Charloteaux, A. Thomas, R. Brasseur, Protein-nucleic acid recognition: statistical analy- sis of atomic interactions and influence of DNA structure, Proteins 61 (2005) 258-271.

[41] M. Arbabi Ghahroudi, A. Desmyter, L. Wyns, R. Hamers, S. Muyldermans, Selection and identification of single domain antibody fragments from camel heavy-chain antibodies, FEBS Lett. 414 (1997) 521-526.

[42] P.Y. Watson, M.J. Fedor, The glmS riboswitch integrates signals from activating and inhibitory metabolites in vivo, Nat. Struct. Mol. Biol. 18 (2011) 359-363.

[43] D.J. Klein, A.R. Ferre-D'Amare, Structural basis of glmS ribozyme activation by glucosamine-6-phosphate, Science 313 (2006) 1752-1756.

[44] M. Abasi, Z. Bazi, S. Mohammadi-Yeganeh, M. Soleimani, V. Haghpanah, N. Zargami, H. Ghanbarian, 7SK small nuclear RNA transcription level down-regulates in human tumors and stem cells, Med. Oncol. 33 (2016) 128.

[45] E.A. Doherty, J.A. Doudna, The P4-P6 domain directs higher order folding of the tetrahymena ribozyme core, Biochemistry 36 (1997) 3159-3169.

[46] M. Szabat, T. Pedzinski, T. Czapik, E. Kierzek, R. Kierzek, Structural aspects of the antiparallel and parallel duplexes formed by DNA, 2'-O-methyl RNA and RNA oligonucleotides, PLoS One 10 (2015), e0143354.

[47] M. Dumoulin, K. Conrath, A. Van Meirhaeghe, F. Meersman, K. Heremans, L.G. Frenken, S. Muyldermans, L. Wyns, A. Matagne, Single-domain antibody fragments with high conformational stability, Protein Sci. 11 (2002) 500-515.

[48] B.T. Andrews, D.T. Capraro, J.I. Sulkowska, J.N. Onuchic, P.A. Jennings, Hysteresis as a marker for complex, overlapping landscapes in proteins, J. Phys. Chem. Lett. 4 (2013) 180-188.

[49] F. Michel, Hysteresis and partial irreversibility of denaturation of DNA as a means of investigating the topology of base distribution constraints: application to a yeast rho- (petite) mitochondrial DNA, J. Mol. Biol. 89 (1974) 305-326.

[50] A.P. Russell, D.S. Holleman, The thermal denaturation of DNA: average length and composition of denatured areas, Nucleic Acids Res. 1 (1974) 959-978.

[51] E.M. Sherman, S. Holmes, J.D. Ye, Specific RNA-binding antibodies with a four-amino-acid code, J. Mol. Biol. 426 (2014) 2145-2157.

[52] Y. Koldobskaya, E.M. Duguid, D.M. Shechner, N.B. Suslov, J. Ye, S.S. Sidhu, D.P. Bartel, S. Koide, A.A. Kossiakoff, J.A. Piccirilli, A portable RNA sequence whose recognition by a synthetic antibody facilitates structural determination, Nat. Struct. Mol. Biol. 18 (2011) 100-106.

[53] Y. Shao, H. Huang, D. Qin, N.S. Li, A. Koide, J.P. Staley, S. Koide, A.A. Kossiakoff, J.A. Piccirilli, Specific recognition of a single-stranded RNA sequence by a synthetic antibody fragment, J. Mol. Biol. 428 (2016) 4100-4114.

[54] C. Low, Y.H. Yau, E. Pardon, C. Jegerschold, L. Wahlin, E.M. Quistgaard, P. Moberg, S. Geifman-Shochat, J. Steyaert, P. Nordlund, Nanobody mediated crystallization of an archeal mechanosensitive channel, PLoS One 8 (2013), e77984.

[55] Y. Hu, E. Romao, D. Vertommen, C. Vincke, F. MoralesYanez, C. Gutierrez, C. Liu, S. Muyldermans, Generation of Nanobodies against SlyD and development of tools to eliminate this bacterial contaminant from recombinant proteins, Protein Expr. Purif. 137 (2017) 64-76.

[56] A. Skerra, A. Pluckthun, Assembly of a functional immunoglobulin Fv fragment in Escherichia coli, Science 240 (1988) 1038-1041. 